\title{
Killing symmetries of generalized Minkowski spaces. 2- Finite structure of space-time rotation groups in four dimensions
}

\author{
Fabio Cardone ${ }^{a, b}$, Alessio Marrani ${ }^{c}$ and \\ Roberto Mignani ${ }^{c, d}$ \\ $a$ Istituto per lo Studio dei Materiali Nanostrutturati \\ (ISMN-CNR) \\ Via dei Taurini, 19 \\ 00185 ROMA, Italy \\ $b$ I.N.D.A.M. - G.N.F.M. \\ $c$ Dipartimento di Fisica "E. Amaldi" \\ Università degli Studi "Roma Tre" \\ Via della Vasca Navale, 84 \\ 00146 ROMA, Italy \\ $d$ I.N.F.N. - Sezione di Roma III
}

July 14, 2018

\begin{abstract}
In this paper, we continue the study of the Killing symmetries of a N-dimensional generalized Minkowski space, i.e. a space endowed with a (in general non-diagonal) metric tensor, whose coefficients do depend on a set of non-metrical coordinates. We discuss here the finite structure of the space-time rotations in such spaces, by confining ourselves (without loss of generality) to the four-dimensional
\end{abstract}


case. In particular, the results obtained are specialized to the case of a "deformed" Minkowski space $\widetilde{M_{4}}$ (i.e. a pseudoeuclidean space with metric coefficients depending on energy), for which we derive the explicit general form of the finite rotations and boosts in different parametric bases.

\section{INTRODUCTION}

In a previous paper [1], we started the study of the Killing symmetries of a $N$-dimensional generalized Minkowski space, i.e. a space endowed with a (in general non-diagonal) metric tensor, whose coefficients do depend on a set of non-metrical coordinates. In particular, we discussed the infinitesimal-

algebraic structure of the space-time rotations in such a space.

An example of a generalized Minkowski space is provided by the deformed space-time $\widetilde{M}_{4}$ of Deformed Special Relativity (DSR). DSR is a generalization of the standard Special Relativity (SR) based on a "deformation" of spacetime, assumed to be endowed with a metric whose coefficients depend on the energy of the process considered [2]. Such a formalism applies in principle to all four interactions (electromagnetic, weak, strong and gravitational) — at least as far as their nonlocal behavior and nonpotential part is concerned and provides a metric representation of them (at least for the process and in the energy range considered) ([3]-[6]). Moreover, it was shown that DSR is actually a five-dimensional scheme, in the sense that the deformed Minkowski space can be naturally embedded in a larger Riemannian manifold, with 
energy as fifth dimension [7].

In this paper, following the line of mathematical-formal research strated by [1], [10] and [13], we shall continue our investigation by discussing the finite structure of the space-time rotations in generalized Minkowski spaces.

For simplicity's sake, we shall restrict us (without loss of generality) to the four-dimensional case, by specializing our results to the deformed space-time $\widetilde{M_{4}}$ of DSR.

The organization of the paper is as follows. In Sect. 2 we briefly review the formalism of DSR and of the deformed Minkowski space $\widetilde{M}_{4}$. The re-

sults obtained in [1], concerning the maximal Killing group of generalized

$N$-dimensional Minkowski spaces, are summarized in Subsect. 3.1, where we also give the general form of the finite spacetime rotations in terms of the infinitesimal generators of the $N$-d. generalized, homogeneous Lorentz group. In Subsect. 3.2 the latter results are specialized to the case of the deformed, homogeneous Lorentz group $S O(3,1)_{D E F}$. of DSR. Sect. 4 deals with the finite deformed spacetime rotations about a coordinate axis. The explicit form of the infinitesimal generators in the DSR case is given in Subsect. 4.1. Subsections 4.2 and 4.3 discuss finite deformed 3-d. rotations about a coordinate axis and finite deformed boosts along a coordinate axis, respectively. Sect. 5 generalizes the latter results to the case of a generic direction. Finally, Sect. 6 concludes the paper. 


\section{DEFORMED SPECIAL RELATIVITY IN FOUR DIMENSIONS (DSR4)}

The generalized ("deformed") Minkowski space $\widetilde{M_{4}}$ (DMS4) of DSR4 1 is defined as a space with the same local coordinates $x$ of $M_{4}$ (the four-vectors of the usual Minkowski space), but with metric given by the metric tensor ${ }^{2}$

$$
\begin{gathered}
g_{\mu \nu, D S R 4}\left(x^{5}\right)=\operatorname{diag}\left(b_{0}^{2}\left(x^{5}\right),-b_{1}^{2}\left(x^{5}\right),-b_{2}^{2}\left(x^{5}\right),-b_{3}^{2}\left(x^{5}\right)\right)= \\
\stackrel{\mathrm{ESC}}{=}{ }^{\text {off }} \delta_{\mu \nu}\left[b_{0}^{2}\left(x^{5}\right) \delta_{\mu 0}-b_{1}^{2}\left(x^{5}\right) \delta_{\mu 1}-b_{2}^{2}\left(x^{5}\right) \delta_{\mu 2}-b_{3}^{2}\left(x^{5}\right) \delta_{\mu 3}\right],
\end{gathered}
$$

where the $\left\{b_{\mu}^{2}\left(x^{5}\right)\right\}$ are dimensionless, real, positive functions of the independent,

non-metrical (n.m.) variable $x^{5}{ }^{3}$. The generalized interval in $\widetilde{M_{4}}$ is therefore given by $\left(x^{\mu}=\left(x^{0}, x^{1}, x^{2}, x^{3}\right)=(c t, x, y, z)\right.$, with $c$ being the usual light speed in vacuum)

$$
\begin{gathered}
d s^{2}\left(x^{5}\right)=b_{0}^{2}\left(x^{5}\right) c^{2} d t^{2}-\left(b_{1}^{2}\left(x^{5}\right) d x^{2}+b_{2}^{2}\left(x^{5}\right) d y^{2}+b_{3}^{2}\left(x^{5}\right) d z^{2}\right)= \\
=g_{\mu \nu, D S R 4}\left(x^{5}\right) d x^{\mu} d x^{\nu}=d x * d x .
\end{gathered}
$$

\footnotetext{
${ }^{1}$ In the following, we shall use the more explicit notation DSR4 instead of DSR, in order to stress the dimensionality of the spacetime involved, and to distinguish it from the 5-d. Deformed Relativity (DR5), i.e. the embedding of DSR in a 5-dimensional, truly Riemannian, manifold [7].

${ }^{2}$ In the following, Greek indices vary in the range $\{0,1,2,3\}, 3$-vectors are denoted in bold and the notation "ESC on" ("ESC off") means that the Einstein sum convention on repeated indices is (is not) understood.

${ }^{3}$ Such a coordinate is to be interpreted as the energy (see Refs. [3]-[7]); moreover, the index 5 explicitly refers to the above-mentioned fact that the deformed Minkowski space can be "naturally" embedded in a 5-dimensional (Riemannian) space [7].
} 
The last step in $(2)$ defines the $\left(x^{5}\right.$-dependent) scalar product $*$ in the deformed Minkowski space $\widetilde{M}_{4}$. In order to emphasize the dependence of

DMS4 on the variable $x^{5}$, we shall sometimes use the notation $\widetilde{M}_{4}\left(x^{5}\right)$. It follows immediately that $\widetilde{M}_{4}\left(x^{5}\right)$ can be regarded as a particular case of a Riemann space with null curvature.

From the general condition

$$
g_{\mu \nu, D S R 4}\left(x^{5}\right) g_{D S R 4}^{\nu \rho}\left(x^{5}\right)=\delta_{\mu}{ }^{\rho}
$$

we get for the contravariant components of the metric tensor

$$
\begin{gathered}
g_{D S R 4}^{\mu \nu}\left(x^{5}\right)=\operatorname{diag}\left(b_{0}^{-2}\left(x^{5}\right),-b_{1}^{-2}\left(x^{5}\right),-b_{2}^{-2}\left(x^{5}\right),-b_{3}^{-2}\left(x^{5}\right)\right)= \\
\stackrel{\mathrm{ESC}}{=}{ }^{\text {off }} \delta^{\mu \nu}\left(b_{0}^{-2}\left(x^{5}\right) \delta^{\mu 0}-b_{1}^{-2}\left(x^{5}\right) \delta^{\mu 1}-b_{2}^{-2}\left(x^{5}\right) \delta^{\mu 2}-b_{3}^{-2}\left(x^{5}\right) \delta^{\mu 3}\right) .
\end{gathered}
$$

Let us stress that metric (1) is supposed to hold at a local (and not global) scale. We shall therefore refer to it as a "topical" deformed metric, because it is supposed to be valid not everywhere, but only in a suitable (local) space-time region (characteristic of both the system and the interaction considered). In brief, DSR4 can be regarded as a (local) "anisotropizing deforming" generalization of SR, synthetically expressed by the metric transition $g_{\mu \nu, S R} \rightarrow g_{\mu \nu, D S R 4}\left(x^{5}\right)$ (with $g_{\mu \nu, S R} \equiv \operatorname{diag}(1,-1,-1,-1)$ ).

The two basic postulates of DSR4 (which generalize those of standard SR) are [2]:

1- Space-time properties: Space-time is homogeneous, but space is not 
necessarily isotropic; a reference frame in which space-time is endowed with such properties is called a "topical" reference frame (TIRF). Two TIRF's are in general moving uniformly with respect to each other (i.e., as in SR, they are connected by a "inertiality" relation, which defines an equivalence class of $\infty^{3}$ TIRF );

2- Generalized Principle of Relativity (or Principle of Metric Invariance): All physical measurements within each TIRF must be carried out via the same metric.

The metric (1) is just a possible realization of the above postulates. We refer the reader to Refs. [3]-[7] for the explicit expressions of the phenomenological energy-dependent metrics for the four fundamental interactions ${ }^{4}$.

\footnotetext{
${ }^{4}$ Since the metric coefficients $b_{\mu}^{2}\left(x^{5}\right)$ are dimensionless, they actually do depend on the ratio $\frac{x^{5}}{x_{0}^{5}}$, where$$
x_{0}^{5} \equiv \ell_{0} E_{0}
$$

is a fundamental length, proportional (by the dimensionally-transposing constant $\ell_{0}$ ) to the threshold energy $E_{0}$, characteristic of the interaction considered (see Refs. [3]-[7]).
} 


\section{GENERAL FORM OF FINITE SPACE- TIME ROTATIONS IN 4-DIMENSIONAL GENERALIZED MINKOWSKI SPACES}

\subsection{Maximal Killing Group of Generalized Minkowski Spaces}

A $N$-dimensional generalized Minkowski space $\widetilde{M_{N}}\left(\{x\}_{n . m .}\right)$ is a Riemann space endowed with the global metric structure [1]

$$
d s^{2}=g_{\mu \nu}\left(\{x\}_{n . m .}\right) d x^{\mu} d x^{\nu}
$$

where the (in general non-diagonal) metric tensor $g_{\mu \nu}\left(\{x\}_{n . m .}\right)(\mu, \nu=1,2, \ldots, N)$ depends on a set $\{x\}_{n . m}$. of $N_{n . m}$. non-metrical coordinates (i.e. different from the $N$ coordinates related to the dimensions of the space considered). We shall assume the (not necessarily hyperbolic) signature $(T, S)$ (i.e. $T$ timelike dimensions and $S=N-T$ spacelike dimensions). It follows that $\widetilde{M_{N}}\left(\{x\}_{n . m .}\right)$ is flat, because all the components of the Riemann-Christoffel tensor vanish.

The maximal Killing group of $\widetilde{M_{N}}\left(\{x\}_{n . m}\right)$ is the generalized Poincaré (or inhomogeneous Lorentz) group $P(S, T)_{G E N \text {. }}^{N(N+1)}$

$$
P(T, S)_{G E N .}^{N(N+1) / 2}=S O(T, S)_{G E N .}^{N(N-1) / 2} \otimes_{s} \operatorname{Tr} .(T, S)_{G E N .}^{N},
$$

i.e. the (semidirect) product of the Lie group of $N$-dimensional space-time rotations (or $N$-d. generalized, homogeneous Lorentz group $S O(T, S)_{G E N .}^{N(N-1) / 2}$ ) with $N(N-1) / 2$ parameters, and of the Lie group of $N$-dimensional spacetime translations $\operatorname{Tr} .(T, S)_{G E N}^{N}$. with $N$ parameters (see Ref. [1]). The semidirect [8] nature of the group product is due to the fact that, as it shall be explicitely derived (in the hyperbolically-signed case $N=4, S=3, T=1$ of DSR4, without loss of generality) in a forthcoming paper [9], in general we have that

$$
\begin{gathered}
\exists \text { at least } 1(\mu, \nu, \rho) \in\{1,2, \ldots, N\}^{3}: \\
:\left[I_{G E N .}^{\mu \nu}\left(\{x\}_{n . m .}\right), \Upsilon_{G E N .}^{\rho}\left(\{x\}_{n . m .}\right)\right] \neq 0, \forall\{x\}_{n . m .},
\end{gathered}
$$


where $I_{G E N .}^{\mu \nu}\left(\{x\}_{n . m .}\right)$ and $\Upsilon_{G E N .}^{\rho}\left(\{x\}_{n . m .}\right)$ are the infinitesimal generators of $S O(T, S)_{G E N .}^{N(N-1) / 2}$ and $\operatorname{Tr} .(T, S)_{G E N .}^{N}$, respectively.

The infinitesimal generators $\left\{\left(I^{\alpha \beta}\right)^{\mu}{ }_{\nu}\left(\{x\}_{n . m .}\right)\right\}_{\alpha, \beta=1 \ldots N}$ of $S O(T, S)_{G E N .}^{N(N-1) / 2}$ satisfy the generalized Lorentz algebra [1]

$$
\begin{gathered}
{\left[I^{\alpha \beta}\left(\{x\}_{n . m .}\right), I^{\rho \sigma}\left(\{x\}_{n . m .}\right)\right]=} \\
=g^{\alpha \sigma}\left(\{x\}_{n . m .}\right) I^{\beta \rho}\left(\{x\}_{n . m .}\right)+g^{\beta \rho}\left(\{x\}_{n . m .}\right) I^{\alpha \sigma}\left(\{x\}_{n . m .}\right)+ \\
-g^{\alpha \rho}\left(\{x\}_{n . m .}\right) I^{\beta \sigma}\left(\{x\}_{n . m .}\right)-g^{\beta \sigma}\left(\{x\}_{n . m .}\right) I^{\alpha \rho}\left(\{x\}_{n . m .}\right) .
\end{gathered}
$$

On the basis of the results of [1], the infinitesimal transformation corresponding, in a 4-d. generalized Minkowski space (with $S(<4)$ spacelike and $T=4-S$ timelike dimensions) to the element $g$ of the generalized,

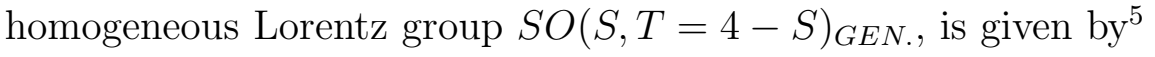




$$
\begin{gathered}
\delta g: x^{\mu} \rightarrow\left(x^{\prime}\right)_{(g)}^{\mu}\left(\{x\}_{m .},\{x\}_{n . m .}\right)=\left(x^{\mu}\right)_{(g)}^{\prime}\left(\{x\}_{m .},\{x\}_{n . m .} .\right)= \\
=x^{\mu}+\delta x_{(g)}^{\mu}\left(\{x\}_{m .},\{x\}_{n . m .}\right)=x^{\mu}+\delta \omega_{\nu}^{\mu}\left(g,\{x\}_{n . m .}\right) x^{\nu}= \\
=x^{\mu}+\frac{1}{2} \delta \omega_{\alpha \beta}(g)\left(I^{\alpha \beta}\right)_{\nu}^{\mu}\left(\{x\}_{n . m .}\right) x^{\nu}= \\
=x^{\mu}+\left(-\theta_{l}(g) S^{l}\left(\{x\}_{n . m .}\right)-\zeta_{i}(g) K^{i}\left(\{x\}_{n . m .}\right)\right)_{\nu}^{\mu} x^{\nu}= \\
=x^{\mu}+\left(-\theta(g) \cdot \mathbf{S}\left(\{x\}_{n . m .}\right)-\zeta(g) \cdot \mathbf{K}\left(\{x\}_{n . m .}\right)\right)_{\nu}^{\mu} x^{\nu}= \\
=\left(1-\theta(g) \cdot \mathbf{S}\left(\{x\}_{n . m .}\right)-\zeta(g) \cdot \mathbf{K}\left(\{x\}_{n . m .}\right)\right)_{\nu}^{\mu} x^{\nu}= \\
=\left(1-\theta_{1}(g) S^{1}\left(\{x\}_{n . m .} .\right)-\theta_{2}(g) S^{2}\left(\{x\}_{n . m .}\right)-\theta_{3}(g) S^{3}\left(\{x\}_{n . m .}\right)+\right. \\
\left.-\zeta_{1}(g) K^{1}\left(\{x\}_{n . m .}\right)-\zeta_{2}(g) K^{2}\left(\{x\}_{n . m .}\right)-\zeta_{3}(g) K^{3}\left(\{x\}_{n . m .}\right)\right)_{\nu}^{\mu} x^{\nu} .
\end{gathered}
$$

Here, 1 is the identity of $S O(S, T=4-S)_{G E N}$, and the completely covariant, antisymmetric 4-tensor $\delta \omega_{\alpha \beta}(g)$ is the tensor of the 4(4-1)/2=6 dimensionless

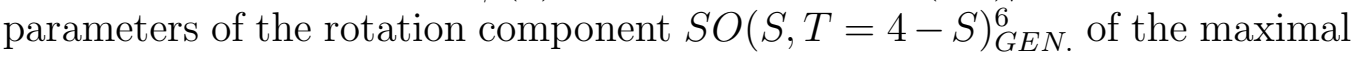

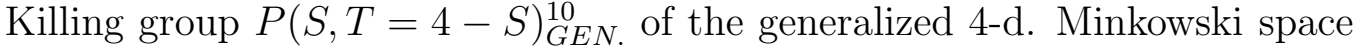
considered. As shown in [1], such a tensor can be expressed as

$$
\delta \omega_{\alpha \beta}(g)=\left(\begin{array}{cccc}
0 & -\zeta^{1}(g) & -\zeta^{2}(g) & -\zeta^{3}(g) \\
\zeta^{1}(g) & 0 & -\theta^{3}(g) & \theta^{2}(g) \\
\zeta^{2}(g) & \theta^{3}(g) & 0 & -\theta^{1}(g) \\
\zeta^{3}(g) & -\theta^{2}(g) & \theta^{1}(g) & 0
\end{array}\right),
$$

where the rotation parameter (Euclidean) axial 3-vector $\theta(g)$ and the boost parameter (Euclidean) polar 3 -vector $\zeta(g)$ are defined respectively by $\left(\epsilon_{i j k}\right.$ is

\footnotetext{
${ }^{5}$ For precision's sake, in general for infinitesimal deformed boost transformations $g \in$ $S O(3,1)_{D E F}$. should be replaced by $\delta g$, where $\delta g \in s u(2)_{D E F} . \times s u(2)_{D E F}$. i.e. it is an element of the deformed Lorentz algebra. But, for simplicity's sake, we will omit, but mean, this cumbersome notation.
} 
the Euclidean Levi-Civita 3 -tensor, with the assumed conbention $\epsilon_{123} \equiv 1$ )

$$
\begin{gathered}
\theta^{i}(g)=\theta_{i}(g) \equiv-\frac{1}{2} \epsilon_{i}^{j k} \delta \omega_{j k}(g), \\
\zeta^{i}(g)=\zeta_{i}(g) \equiv-\delta \omega_{0 i}(g),
\end{gathered}
$$

namely

$$
\begin{aligned}
& \theta(g) \equiv\left(-\delta \omega_{23}(g),-\delta \omega_{31}(g),-\delta \omega_{12}(g)\right), \\
& \zeta(g) \equiv\left(-\delta \omega_{01}(g),-\delta \omega_{02}(g),-\delta \omega_{03}(g)\right) .
\end{aligned}
$$

Moreover, the matrix 3-vectors

$$
\begin{gathered}
\mathbf{S}\left(\{x\}_{n . m .}\right) \equiv\left(I^{23}\left(\{x\}_{n . m .}\right), I^{31}\left(\{x\}_{n . m .}\right), I^{12}\left(\{x\}_{n . m .}\right)\right) \\
\mathbf{K}\left(\{x\}_{n . m .}\right) \equiv\left(I^{01}\left(\{x\}_{n . m .}\right), I^{02}\left(\{x\}_{n . m .}\right), I^{03}\left(\{x\}_{n . m .}\right)\right)
\end{gathered}
$$

constitute the self-representation basis for $S O(S, T=4-S)_{G E N \text {. }}$.

From Eq. (9) it follows that the finite space-time rotation in $\widetilde{M}_{4}\left(\{x\}_{n . m .}\right)$

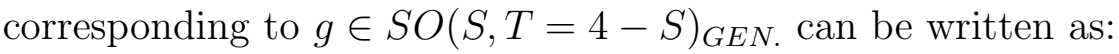

$$
\begin{gathered}
S O(S, T=4-S)_{G E N .} \ni g: \\
x^{\mu} \rightarrow\left(x^{\prime}\right)_{(g)}^{\mu}\left(\{x\}_{m .},\{x\}_{n . m .}\right)=\left(x^{\mu}\right)_{(g)}^{\prime}\left(\{x\}_{m .},\{x\}_{n . m .}\right)= \\
=\exp \left(-\theta(g) \cdot \mathbf{S}\left(\{x\}_{n . m .}\right)-\zeta(g) \cdot \mathbf{K}\left(\{x\}_{n . m .}\right)\right)_{\nu}^{\mu} x^{\nu}= \\
=\exp \left(-\theta_{1}(g) S^{1}\left(\{x\}_{n . m .}\right)-\theta_{2}(g) S^{2}\left(\{x\}_{n . m .}\right)-\theta_{3}(g) S^{3}\left(\{x\}_{n . m .}\right)+\right. \\
\left.-\zeta_{1}(g) K^{1}\left(\{x\}_{n . m .}\right)-\zeta_{2}(g) K^{2}\left(\{x\}_{n . m .}\right)-\zeta_{3}(g) K^{3}\left(\{x\}_{n . m .}\right)\right)_{\nu}^{\mu} x^{\nu}
\end{gathered}
$$


or, by a series development of the exponential:

$$
\begin{gathered}
S O(S, T=4-S)_{G E N .} \ni g: \\
x^{\mu} \rightarrow\left(x^{\prime}\right)_{(g)}^{\mu}\left(\{x\}_{m .},\{x\}_{n . m .}\right)=\left(x^{\mu}\right)_{(g)}^{\prime}\left(\{x\}_{m .},\{x\}_{n . m .}\right)= \\
=\left(\sum_{n=0}^{\infty} \frac{1}{n !}\left(-\theta(g) \cdot \mathbf{S}\left(\{x\}_{n . m .}\right)-\zeta(g) \cdot \mathbf{K}\left(\{x\}_{n . m .}\right)\right)^{n}\right)_{\nu}^{\mu} x^{\nu}= \\
=\left(\sum _ { n = 0 } ^ { \infty } \frac { 1 } { n ! } \left(-\theta_{1}(g) S^{1}\left(\{x\}_{n . m .}\right)-\theta_{2}(g) S^{2}\left(\{x\}_{n . m .}\right)-\theta_{3}(g) S^{3}\left(\{x\}_{n . m .}\right)+\right.\right. \\
\left.\left.-\zeta_{1}(g) K^{1}\left(\{x\}_{n . m .}\right)-\zeta_{2}(g) K^{2}\left(\{x\}_{n . m .}\right)-\zeta_{3}(g) K^{3}\left(\{x\}_{n . m .}\right)\right)^{n}\right)_{\nu}^{\mu} x^{\nu} .
\end{gathered}
$$

Since the generalized Lorentz algebra (8) is non-commutative, the group $S O(S, T)_{G E N .}^{N(N-1) / 2}$ is non-Abelian, and therefore the 4-d. finite transformations (17), (18) do not commute.

\subsection{Finite Transformations of the Deformed, Homoge- neous Lorentz Group $S O(3,1)_{D E F}$. of DSR4.}

In the case of the deformed Minkowski space-time $\widetilde{M}_{4}\left(x^{5}\right)$ (corresponding to $\left.N=4, S=3, T=1, x^{\mu}=\left\{x^{0}, x^{1}, x^{2}, x^{3}\right\},\{x\}_{n . m .}=x^{5}\right)$ the generalized Lorentz algebra (8) becomes the (4-d.) deformed Lorentz algebra su $(2)_{D E F .} \times$ 
$s u(2)_{D E F}$. and the infinitesimal space-time rotation (9) reads

$$
\begin{gathered}
s u(2)_{D E F .} \times s u(2)_{D E F .} \ni \delta g: \\
x^{\mu} \rightarrow x_{(g), D S R 4}^{\prime}\left(\{x\}_{m .}, x^{5}\right)=\left(x^{\mu}\right)_{(g), D S R 4}^{\prime}\left(\{x\}_{m .}, x^{5}\right)= \\
=x^{\mu}+\delta x_{(g), D S R 4}^{\mu}\left(\{x\}_{m .}, x^{5}\right)= \\
=x^{\mu}+\left(-\theta_{l}(g) S_{D S R 4}^{l}\left(x^{5}\right)-\zeta_{i}(g) K_{D S R 4}^{i}\left(x^{5}\right)\right)_{\nu}^{\mu} x^{\nu}= \\
=x^{\mu}+\left(-\theta(g) \cdot \mathbf{S}_{D S R 4}\left(x^{5}\right)-\zeta(g) \cdot \mathbf{K}_{D S R 4}\left(x^{5}\right)\right)_{\nu}^{\mu} x^{\nu}= \\
=\left(1-\theta(g) \cdot \mathbf{S}_{D S R 4}\left(x^{5}\right)-\zeta(g) \cdot \mathbf{K}_{D S R 4}\left(x^{5}\right)\right)_{\nu}^{\mu} x^{\nu}= \\
=\left(1-\theta_{1}(g) S_{D S R 4}^{1}\left(x^{5}\right)-\theta_{2}(g) S_{D S R 4}^{2}\left(x^{5}\right)-\theta_{3}(g) S_{D S R 4}^{3}\left(x^{5}\right)+\right. \\
\left.-\zeta_{1}(g) K_{D S R 4}^{1}\left(x^{5}\right)-\zeta_{2}(g) K_{D S R 4}^{2}\left(x^{5}\right)-\zeta_{3}(g) K_{D S R 4}^{3}\left(x^{5}\right)\right)_{\nu}^{\mu} x^{\nu},
\end{gathered}
$$

with obvious meaning of notation (in particular 1 denotes the identity of $S O(3,1)_{D E F}$. corresponding -by the homomorphic exponential mapping- to the origin of the algebra $\left.s u(2)_{D E F} . \times s u(2)_{D E F}.\right)$.

In this case (like in the standard $\mathrm{SR}$ ), since the couple $(S, T)=(3,1)$ denoting the hyperbolic metric signature of $\widetilde{M}_{4}\left(x^{5}\right)$ is fixed, it is possible to give a physical interpretation of parameters and generators: $\theta(g)$ and $\zeta(g)$ are, respectively, the deformed rotation and boost Euclidean 3-vectors, whereas $\mathbf{S}_{D S R 4}\left(x^{5}\right)$ and $\mathbf{K}_{D S R 4}\left(x^{5}\right)$ are the (operatorial 3-vectors of) generators of the corresponding transformations of the deformed, homogeneous Lorentz group $S O(3,1)_{D E F}$., satisfying the (4-d.) deformed Lorentz algebra 
$s u(2)_{D E F .} \times s u(2)_{D E F .}:$

$$
\left\{\begin{array}{l}
{\left[S_{D S R 4}^{i}\left(x^{5}\right), S_{D S R 4}^{j}\left(x^{5}\right)\right]=\epsilon_{i j k} b_{k}^{-2}\left(x^{5}\right) S_{D S R 4}^{k}\left(x^{5}\right) ;} \\
{\left[K_{D S R 4}^{i}\left(x^{5}\right), K_{D S R 4}^{j}\left(x^{5}\right)\right]=-b_{0}^{-2}\left(x^{5}\right) \epsilon_{i j k} S_{D S R 4}^{k}\left(x^{5}\right) ;} \\
{\left[S_{D S R 4}^{i}\left(x^{5}\right), K_{D S R 4}^{j}\left(x^{5}\right)\right]=\epsilon_{i j l} b_{j}^{-2} K_{D S R 4}^{l}\left(x^{5}\right) .}
\end{array}\right.
$$

In the DSR4 case, Eq. (18) for a finite transformation becomes

$$
\begin{gathered}
S O(3,1)_{D E F .} \ni g: \\
x^{\mu} \rightarrow\left(x^{\prime}\right)_{(g), D S R 4}^{\mu}\left(\{x\}_{m .}, x^{5}\right)=\left(x^{\mu}\right)_{(g), D S R 4}^{\prime}\left(\{x\}_{m .}, x^{5}\right)= \\
=\exp \left(-\theta(g) \cdot \mathbf{S}_{D S R 4}\left(x^{5}\right)-\zeta(g) \cdot \mathbf{K}_{D S R 4}\left(x^{5}\right)\right)_{\nu}^{\mu} x^{\nu}= \\
=\exp \left(-\theta_{1}(g) S_{D S R 4}^{1}\left(x^{5}\right)-\theta_{2}(g) S_{D S R 4}^{2}\left(x^{5}\right)-\theta_{3}(g) S_{D S R 4}^{3}{ }^{2}\left(x^{5}\right)+\right. \\
\left.-\zeta_{1}(g) K_{D S R 4}^{1}\left(x^{5}\right)-\zeta_{2}(g) K_{D S R 4}^{2}\left(x^{5}\right)-\zeta_{3}(g) K_{D S R 4}^{3}\left(x^{5}\right)\right)^{\mu}{ }_{\nu} x^{\nu}= \\
\left.=\left(\sum_{n=0}^{\infty} \frac{1}{n !}\left(-\theta(g) \cdot \mathbf{S}_{D S R 4}\left(x^{5}\right)-\zeta(g) \cdot \mathbf{K}_{D S R 4}\left(x^{5}\right)\right)\right)^{n}\right)^{\mu}{ }_{\nu} x^{\nu}= \\
=\left(\sum _ { n = 0 } ^ { \infty } \frac { 1 } { n ! } \left(-\theta_{1}(g) S_{D S R 4}^{1}\left(x^{5}\right)-\theta_{2}(g) S_{D S R 4}^{2}\left(x^{5}\right)-\theta_{3}(g) S_{D S R 4}^{3}\left(x^{5}\right)+\right.\right. \\
\left.\left.-\zeta_{1}(g) K_{D S R 4}^{1}\left(x^{5}\right)-\zeta_{2}(g) K_{D S R 4}^{2}\left(x^{5}\right)-\zeta_{3}(g) K_{D S R 4}^{3}\left(x^{5}\right)\right)^{n}\right)_{\nu}^{\mu} x^{\nu} .
\end{gathered}
$$




\section{FINITE SPACE-TIME ROTATIONS ABOUT A COORDINATE AXIS IN $\widetilde{M}_{4}\left(x^{5}\right)$}

\subsection{Infinitesimal Generators}

Let us recall the explicit form of the matrices of the 4-d. representation of the infinitesimal generators of the group $S O(3,1)_{D E F}$, derived in [1]:

$$
\begin{aligned}
& I_{D S R 4}^{10}\left(x^{5}\right)=\left(\begin{array}{cccc}
0 & -b_{0}^{-2}\left(x^{5}\right) & 0 & 0 \\
-b_{1}^{-2}\left(x^{5}\right) & 0 & 0 & 0 \\
0 & 0 & 0 & 0 \\
0 & 0 & 0 & 0
\end{array}\right) \\
& I_{D S R 4}^{20}\left(x^{5}\right)=\left(\begin{array}{cccc}
0 & 0 & -b_{0}^{-2}\left(x^{5}\right) & 0 \\
0 & 0 & 0 & 0 \\
-b_{2}^{-2}\left(x^{5}\right) & 0 & 0 & 0 \\
0 & 0 & 0 & 0
\end{array}\right) ; \\
& I_{D S R 4}^{30}\left(x^{5}\right)=\left(\begin{array}{cccc}
0 & 0 & 0 & -b_{0}^{-2}\left(x^{5}\right) \\
0 & 0 & 0 & 0 \\
0 & 0 & 0 & 0 \\
-b_{3}^{-2}\left(x^{5}\right) & 0 & 0 & 0
\end{array}\right) ; \\
& I_{D S R 4}^{12}\left(x^{5}\right)=\left(\begin{array}{cccc}
0 & 0 & 0 & 0 \\
0 & 0 & -b_{1}^{-2}\left(x^{5}\right) & 0 \\
0 & b_{2}^{-2}\left(x^{5}\right) & 0 & 0 \\
0 & 0 & 0 & 0
\end{array}\right) \\
& I_{D S R 4}^{23}\left(x^{5}\right)=\left(\begin{array}{cccc}
0 & 0 & 0 & 0 \\
0 & 0 & 0 & 0 \\
0 & 0 & 0 & -b_{2}^{-2}\left(x^{5}\right) \\
0 & 0 & b_{3}^{-2}\left(x^{5}\right) & 0
\end{array}\right)
\end{aligned}
$$




$$
I_{D S R 4}^{31}\left(x^{5}\right)=\left(\begin{array}{cccc}
0 & 0 & 0 & 0 \\
0 & 0 & 0 & b_{1}^{-2}\left(x^{5}\right) \\
0 & 0 & 0 & 0 \\
0 & -b_{3}^{-2}\left(x^{5}\right) & 0 & 0
\end{array}\right) .
$$

In the following, we shall need the (not maximally-ranked) $4 \times 4$ matrices $A_{i}, B_{i}(i=1,2,3)$, defined by:

$$
\begin{aligned}
& A_{1} \equiv\left(\begin{array}{llll}
1 & 0 & 0 & 0 \\
0 & 1 & 0 & 0 \\
0 & 0 & 0 & 0 \\
0 & 0 & 0 & 0
\end{array}\right) ; \\
& A_{2} \equiv\left(\begin{array}{llll}
1 & 0 & 0 & 0 \\
0 & 0 & 0 & 0 \\
0 & 0 & 1 & 0 \\
0 & 0 & 0 & 0
\end{array}\right) ; \\
& A_{3} \equiv\left(\begin{array}{llll}
1 & 0 & 0 & 0 \\
0 & 0 & 0 & 0 \\
0 & 0 & 0 & 0 \\
0 & 0 & 0 & 1
\end{array}\right) ; \\
& B_{1} \equiv\left(\begin{array}{llll}
0 & 0 & 0 & 0 \\
0 & 0 & 0 & 0 \\
0 & 0 & 1 & 0 \\
0 & 0 & 0 & 1
\end{array}\right) ; \\
& B_{2} \equiv\left(\begin{array}{llll}
0 & 0 & 0 & 0 \\
0 & 1 & 0 & 0 \\
0 & 0 & 0 & 0 \\
0 & 0 & 0 & 1
\end{array}\right) ; \\
& B_{3} \equiv\left(\begin{array}{llll}
0 & 0 & 0 & 0 \\
0 & 1 & 0 & 0 \\
0 & 0 & 1 & 0 \\
0 & 0 & 0 & 0
\end{array}\right)
\end{aligned}
$$


It is easy to see, by explicit calculation, that the powers of the generators of deformed boosts and rotations satisfy, respectively, the relations (ESC off) :

$$
\left\{\begin{array}{l}
\left(I_{D S R 4}^{i 0}\left(x^{5}\right)\right)^{0}=1_{4-d .} \\
\left(I_{D S R 4}^{i 0}\left(x^{5}\right)\right)^{2 n}=b_{0}^{-2 n}\left(x^{5}\right) b_{i}^{-2 n}\left(x^{5}\right) A_{i}, n \in N \\
\left(I_{D S R 4}^{i 0}\left(x^{5}\right)\right)^{2 n+1}=b_{0}^{-2 n}\left(x^{5}\right) b_{i}^{-2 n}\left(x^{5}\right) I_{D S R 4}^{i 0}\left(x^{5}\right), n \in N \cup\{0\}
\end{array}\right.
$$

$$
\left\{\begin{array}{l}
\left(I_{D S R 4}^{i j}\left(x^{5}\right)\right)^{0}=1_{4-d .} \\
\left(I_{D S R 4}^{i j}\left(x^{5}\right)\right)^{2 n}=(-1)^{n} b_{i}^{-2 n}\left(x^{5}\right) b_{j}^{-2 n}\left(x^{5}\right) B_{s \neq i, s \neq j}= \\
=(-1)^{n} b_{i}^{-2 n}\left(x^{5}\right) b_{j}^{-2 n}\left(x^{5}\right)\left(\sum_{s=1}^{3}\left(1-\delta_{s i}\right)\left(1-\delta_{s j}\right) B_{s}\right)= \\
\operatorname{ESC} \text { off on } i \text { and } j, \mathrm{ESC} \text { on on } k \\
=1)^{n} b_{i}^{-2 n}\left(x^{5}\right) b_{j}^{-2 n}\left(x^{5}\right)\left|\epsilon_{i j k}\right| B_{k}, n \in N ; \\
\left(I_{D S R 4}^{i j}\left(x^{5}\right)\right)^{2 n+1}=(-1)^{n} b_{i}^{-2 n}\left(x^{5}\right) b_{j}^{-2 n}\left(x^{5}\right) I_{D S R 4}^{i j}\left(x^{5}\right), n \in N \cup\{0\}
\end{array}\right.
$$

$\left(i=1,2,3\right.$, with $1_{4-d}$. being the identity $4 \times 4$ matrix and $\left.\left|\epsilon_{i j k}\right| \equiv \operatorname{sgn}\left(\epsilon_{i j k}\right) \epsilon_{i j k}\right)$. Notice that Eqs. (34) and (35), although obtained by utilizing a 4-d. representation of the infinitesimal generators of $S O(3,1)_{D E F}$. hold true in general at abstract group level (i.e. they are representation-independent).

\subsection{Finite Deformed Boost Along a Coordinate Axis}

Let us first consider a finite deformed boost transformation, with rapidity parameter $\zeta_{1}(g)$ along $\widehat{x^{1}}$. Recalling that $K_{D S R 4}^{i}\left(x^{5}\right) \equiv I_{D S R 4}^{0 i}\left(x^{5}\right) \forall i=1,2,3$, we get, from Eq. (21): 
$S O(3,1)_{D E F .} \ni g:$

$$
\begin{aligned}
& x^{\mu} \rightarrow\left(x^{\prime}\right)_{(g), D S R 4}^{\mu}\left(\{x\}_{m .}, x^{5}\right)=\left(x^{\mu}\right)_{(g), D S R 4}^{\prime}\left(\{x\}_{m .}, x^{5}\right)= \\
& =\exp \left(-\zeta_{1}(g) K_{D S R 4}^{1}\left(x^{5}\right)\right)^{\mu}{ }_{\nu} x^{\nu}=\exp \left(\zeta_{1}(g) I_{D S R 4}^{10}\left(x^{5}\right)\right)^{\mu}{ }_{\nu} x^{\nu}= \\
& =\left(\sum_{n=0}^{\infty} \frac{1}{n !}\left(\zeta_{1}(g) I_{D S R 4}^{10}\left(x^{5}\right)\right)^{n}\right)_{\nu}^{\mu} x^{\nu}=\left(\sum_{n=0}^{\infty} \frac{1}{n !}\left(\zeta_{1}(g)\right)^{n}\left(I_{D S R 4}^{10}\left(x^{5}\right)\right)^{n}\right)_{\nu}^{\mu} x^{\nu}= \\
& =\left(1_{4-d .}+\sum_{n=1}^{\infty} \frac{1}{(2 n) !}\left(\zeta_{1}(g)\right)^{2 n}\left(I_{D S R 4}^{10}\left(x^{5}\right)\right)^{2 n}+\right. \\
& \left.+\sum_{n=0}^{\infty} \frac{1}{(2 n+1) !}\left(\zeta_{1}(g)\right)^{2 n+1}\left(I_{D S R 4}^{10}\left(x^{5}\right)\right)^{2 n+1}\right)_{\nu}^{\mu} x^{\nu}= \\
& =\left(1_{4-d .}+A_{1} \sum_{n=1}^{\infty} \frac{1}{(2 n) !} b_{0}^{-2 n}\left(x^{5}\right) b_{1}^{-2 n}\left(x^{5}\right)\left(\zeta_{1}(g)\right)^{2 n}+\right. \\
& \left.+I_{D S R 4}^{10}\left(x^{5}\right) \sum_{n=0}^{\infty} \frac{1}{(2 n+1) !} b_{0}^{-2 n}\left(x^{5}\right) b_{1}^{-2 n}\left(x^{5}\right)\left(\zeta_{1}(g)\right)^{2 n+1}\right)_{\nu}^{\mu} x^{\nu}= \\
& =\left(1_{4-d .}+A_{1} \sum_{n=1}^{\infty} \frac{1}{(2 n) !}\left(\zeta_{1}(g) b_{0}^{-1}\left(x^{5}\right) b_{1}^{-1}\left(x^{5}\right)\right)^{2 n}+\right. \\
& \left.+I_{D S R 4}^{10}\left(x^{5}\right) b_{0}\left(x^{5}\right) b_{1}\left(x^{5}\right) \sum_{n=0}^{\infty} \frac{1}{(2 n+1) !}\left(\zeta_{1}(g) b_{0}^{-1}\left(x^{5}\right) b_{1}^{-1}\left(x^{5}\right)\right)^{2 n+1}\right)_{\nu}^{\mu} x^{\nu}= \\
& =\left(1_{4-d .}+\left(\left(\cosh \zeta_{1}(g) b_{0}^{-1}\left(x^{5}\right) b_{1}^{-1}\left(x^{5}\right)\right)-1\right) A_{1}+\right. \\
& \left.+b_{0}\left(x^{5}\right) b_{1}\left(x^{5}\right)\left(\sinh \zeta_{1}(g) b_{0}^{-1}\left(x^{5}\right) b_{1}^{-1}\left(x^{5}\right)\right) I_{D S R 4}^{10}\left(x^{5}\right)\right)_{\nu}^{\mu} x^{\nu},
\end{aligned}
$$


where in the last passage the series expansions of hyperbolic functions have been used.

By defining the $4 \times 4$ matrix

$$
\begin{gathered}
\text { Boost }_{D S R 4, \widehat{x^{1}}}\left(g, x^{5}\right) \equiv 1_{4-d .}+\left(\left(\cosh \zeta_{1}(g) b_{0}^{-1}\left(x^{5}\right) b_{1}^{-1}\left(x^{5}\right)\right)-1\right) A_{1}+ \\
+b_{0}\left(x^{5}\right) b_{1}\left(x^{5}\right)\left(\sinh \zeta_{1}(g) b_{0}^{-1}\left(x^{5}\right) b_{1}^{-1}\left(x^{5}\right)\right) I_{D S R 4}^{10}\left(x^{5}\right)
\end{gathered}
$$

Eq. (36) can be rewritten as:

$$
\begin{gathered}
S O(3,1)_{D E F .} \ni g: x^{\mu} \rightarrow\left(x^{\prime}\right)_{(g), D S R 4}^{\mu}\left(\{x\}_{m .}, x^{5}\right)=\left(x^{\mu}\right)_{(g), D S R 4}^{\prime}\left(\{x\}_{m .}, x^{5}\right)= \\
=\left(\text { Boost }_{D S R 4, \widehat{x^{1}}}\left(g, x^{5}\right)\right)_{\nu}^{\mu} x^{\nu} .
\end{gathered}
$$

We find therefore

$$
\begin{gathered}
\left(\begin{array}{c}
\left(x^{\prime}\right)_{(g), D S R 4}^{0}\left(\{x\}_{m .}, x^{5}\right) \\
\left(x^{\prime}\right)_{(g), D S R 4}^{1}\left(\{x\}_{m .}, x^{5}\right) \\
\left(x^{\prime}\right)_{(g), D S R 4}^{2}\left(\{x\}_{m .}, x^{5}\right) \\
\left(x^{\prime}\right)_{(g), D S R 4}^{3}\left(\{x\}_{m .}, x^{5}\right)
\end{array}\right)=\left(\begin{array}{c}
\left(x^{0}\right)_{(g), D S R 4}^{\prime}\left(\{x\}_{m .}, x^{5}\right) \\
\left(x^{1}\right)_{(g), D S R 4}^{\prime}\left(\{x\}_{m .}, x^{5}\right) \\
\left(x^{2}\right)_{(g), D S R 4}^{\prime}\left(\{x\}_{m .}, x^{5}\right) \\
\left(x^{3}\right)_{(g), D S R 4}^{\prime}\left(\{x\}_{m .}, x^{5}\right)
\end{array}\right)= \\
=\left(\text { Boost }_{D S R 4, \widehat{x^{1}}}\left(g, x^{5}\right)\right) \times\left(\begin{array}{c}
x^{0} \\
x^{1} \\
x^{2} \\
x^{3}
\end{array}\right)=
\end{gathered}
$$$$
=\left(\begin{array}{c}
\left(\cosh \zeta_{1}(g) b_{0}^{-1}\left(x^{5}\right) b_{1}^{-1}\left(x^{5}\right)\right) x^{0}-b_{0}^{-1}\left(x^{5}\right) b_{1}\left(x^{5}\right)\left(\sinh \zeta_{1}(g) b_{0}^{-1}\left(x^{5}\right) b_{1}^{-1}\left(x^{5}\right)\right) x^{1} \\
-b_{0}\left(x^{5}\right) b_{1}^{-1}\left(x^{5}\right)\left(\sinh \zeta_{1}(g) b_{0}^{-1}\left(x^{5}\right) b_{1}^{-1}\left(x^{5}\right)\right) x^{0}+\left(\cosh \zeta_{1}(g) b_{0}^{-1}\left(x^{5}\right) b_{1}^{-1}\left(x^{5}\right)\right) x^{1} \\
x^{2} \\
x^{3}
\end{array}\right) .
$$ 
Analogous results are obtained for the finite deformed boosts along the other two spatial axes. By defining the matrices

$$
\begin{aligned}
& \text { Boost }_{D S R 4, \widehat{x^{2}}}\left(g, x^{5}\right) \equiv 1_{4-d .}+\left(\left(\cosh \zeta_{2}(g) b_{0}^{-1}\left(x^{5}\right) b_{2}^{-1}\left(x^{5}\right)\right)-1\right) A_{2}+ \\
& +b_{0}\left(x^{5}\right) b_{2}\left(x^{5}\right)\left(\sinh \zeta_{2}(g) b_{0}^{-1}\left(x^{5}\right) b_{2}^{-1}\left(x^{5}\right)\right) I_{D S R 4}^{20}\left(x^{5}\right) ; \\
& \text { Boost }_{D S R 4, \widehat{x^{3}}}\left(g, x^{5}\right) \equiv 1_{4-d .}+\left(\left(\cosh \zeta_{3}(g) b_{0}^{-1}\left(x^{5}\right) b_{3}^{-1}\left(x^{5}\right)\right)-1\right) A_{3}+ \\
& +b_{0}\left(x^{5}\right) b_{3}\left(x^{5}\right)\left(\sinh \zeta_{3}(g) b_{0}^{-1}\left(x^{5}\right) b_{3}^{-1}\left(x^{5}\right)\right) I_{D S R 4}^{30}\left(x^{5}\right)
\end{aligned}
$$

we get indeed

$$
\begin{aligned}
& S O(3,1)_{D E F .} \ni g: x^{\mu} \rightarrow\left(x^{\prime}\right)_{(g), D S R 4}^{\mu}\left(\{x\}_{m .}, x^{5}\right)=\left(x^{\mu}\right)_{(g), D S R 4}^{\prime}\left(\{x\}_{m .}, x^{5}\right)= \\
&=\left(\text { Boost }_{D S R 4, \widehat{x^{2}}}\left(g, x^{5}\right)\right)_{\nu}^{\mu} x^{\nu} ; \\
& S O(3,1)_{D E F .} \ni g: x^{\mu} \rightarrow\left(x^{\prime}\right)_{(g), D S R 4}^{\mu}\left(\{x\}_{m .}, x^{5}\right)=\left(x^{\mu}\right)_{(g), D S R 4}^{\prime}\left(\{x\}_{m .}, x^{5}\right)= \\
&=\left(\text { Boost }_{D S R 4, \widehat{x^{3}}}\left(g, x^{5}\right)\right)_{\nu}^{\mu} x^{\nu} .
\end{aligned}
$$

Let us introduce the (effective) deformed rapidity $\tilde{\zeta}\left(g, x^{5}\right)$, defined by

$$
\tilde{\zeta}_{i}\left(g, x^{5}\right) \equiv \zeta_{i}(g) b_{0}^{-1}\left(x^{5}\right) b_{i}^{-1}\left(x^{5}\right) \quad \forall i=1,2,3
$$


Then, a finite deformed boost transformation, with rapidity parameter $\zeta_{i}(g)$ along $\widehat{x^{i}}$ can be written in compact form as

$$
\begin{gathered}
\quad\left(x^{\prime}\right)_{(g), D S R 4}^{0}\left(\{x\}_{m .}, x^{5}\right)=\left(x^{0}\right)_{(g), D S R 4}^{\prime}\left(\{x\}_{m .}, x^{5}\right)= \\
=\left(\cosh \tilde{\zeta}_{i}\left(g, x^{5}\right)\right) x^{0}-b_{0}^{-1}\left(x^{5}\right) b_{i}\left(x^{5}\right)\left(\sinh \tilde{\zeta}_{i}\left(g, x^{5}\right)\right) x^{i}, \\
\left(x^{\prime}\right)_{(g), D S R 4}^{i}\left(\{x\}_{m .}, x^{5}\right)=\left(x^{i}\right)_{(g), D S R 4}^{\prime}\left(\{x\}_{m .}, x^{5}\right)= \\
=-b_{0}\left(x^{5}\right) b_{i}^{-1}\left(x^{5}\right)\left(\sinh \tilde{\zeta}_{i}\left(g, x^{5}\right)\right) x^{0}+\left(\cosh \tilde{\zeta}_{i}\left(g, x^{5}\right)\right) x^{i}, \\
\left(x^{\prime}\right)_{\substack{g), D S R 4 \\
k \neq i}}\left(\{x\}_{m .}, x^{5}\right)=\left(x^{k \neq i}\right)_{(g), D S R 4}^{\prime}\left(\{x\}_{m .}, x^{5}\right)=x^{k \neq i} .
\end{gathered}
$$

By recalling the expression of a boost in SR(4)

$$
\left\{\begin{array}{l}
\left(x^{\prime}\right)_{(g), S R 4}^{0}\left(\{x\}_{m .}\right)=\left(x^{0}\right)_{(g), S R 4}^{\prime}\left(\{x\}_{m .}\right)=\left(\cosh \zeta_{i}(g)\right) x^{0}-\left(\sinh \zeta_{i}(g)\right) x^{i} \\
\left(x^{\prime}\right)_{(g), S R 4}^{i}\left(\{x\}_{m .}\right)=\left(x^{i}\right)_{(g), S R 4}^{\prime}\left(\{x\}_{m .}\right)=-\left(\sinh \zeta_{i}(g)\right) x^{0}+\left(\cosh \zeta_{i}(g)\right) x^{i} \\
\left(x^{\prime}\right)_{(g), S R 4}^{k \neq i}\left(\{x\}_{m .}\right)=\left(x^{k \neq i}\right)_{(g), S R 4}^{\prime}\left(\{x\}_{m} .\right)=x^{k \neq i},
\end{array}\right.
$$

it is easily seen that the deforming transition SR $\rightarrow$ DSR4 corresponds - at the level of group parameters - to the deforming and anisotropizing rescaling of rapidities $\zeta_{i}(g) \rightarrow \tilde{\zeta}_{i}\left(g, x^{5}\right), \forall i=1,2,3$. 


\subsubsection{Parametric Change of Basis for a Deformed Boost along a Coordinate Axis.}

We recall that a deformed boost with speed parameter $v^{i}$ along $\widehat{x^{i}}$ reads (see Ref. [10]) (ESC off throughout):

$$
\begin{gathered}
\left(x^{\prime}\right)_{(g), D S R 4}^{i}\left(\{x\}_{m .}, x^{5}\right)=\left(x^{i}\right)_{(g), D S R 4}^{\prime}\left(\{x\}_{m .}, x^{5}\right)= \\
=\widetilde{\gamma}(g)\left(x^{i}-v^{i}(g) t\right)=\widetilde{\gamma}(g)\left(x^{i}-\widetilde{\beta}(g) \frac{b_{0}\left(x^{5}\right)}{b_{i}\left(x^{5}\right)} c t\right), \\
\left(x^{\prime}\right)_{(g), D S R 4}^{k \neq i}\left(\{x\}_{m .}, x^{5}\right)=\left(x^{k \neq i}\right)_{(g), D S R 4}^{\prime}\left(\{x\}_{m .}, x^{5}\right)=x^{k \neq i}, \\
=\widetilde{\gamma}(g)\left(t-\frac{v^{i}(g) b_{i}^{2}\left(x^{5}\right)}{c^{2} b_{0}^{2}\left(x^{5}\right)} x^{i}\right)=\widetilde{\gamma}(g)\left(t-\frac{\widetilde{\beta}^{2}(g)}{v^{i}(g)} x^{i}\right)
\end{gathered}
$$

where (the dependence on $x^{5}$ is omitted, but understood)

$$
\begin{gathered}
\widetilde{\beta}(g)=\widetilde{\beta^{i}}(g) \equiv \frac{v^{i}(g) b_{i}\left(x^{5}\right)}{c b_{0}\left(x^{5}\right)} \equiv \frac{v^{i}(g)}{u_{i}} \\
\widetilde{\gamma}(g)=\widetilde{\gamma^{i}}(g) \equiv\left(1-\left(\widetilde{\beta^{i}}(g)\right)^{2}\right)^{-1 / 2}=\left(1-\left(\frac{v^{i}(g) b_{i}\left(x^{5}\right)}{c b_{0}\left(x^{5}\right)}\right)^{2}\right)^{-1 / 2}
\end{gathered}
$$


Quantity $u_{i}$ is the maximal causal speed (along $\widehat{x^{i}}$ ) in $\widetilde{M_{4}}\left(x_{5}\right)$, i.e. the generalization of the speed of light for SR ([2], [10]). In general, we have

$$
\mathbf{u} \equiv\left(c \frac{b_{0}\left(x^{5}\right)}{b_{1}\left(x^{5}\right)} \widehat{x}, c \frac{b_{0}\left(x^{5}\right)}{b_{2}\left(x^{5}\right)} \widehat{y}, c \frac{b_{0}\left(x^{5}\right)}{b_{3}\left(x^{5}\right)} \widehat{z}\right) .
$$

Eq. (47) can be put in symmetrical form with respect to time and space coordinates by introducing the dimensional coordinate $\tilde{x}^{0}$ defined by [10]

$$
\tilde{x}^{0} \equiv u^{i} t=c \frac{b_{0}\left(x^{5}\right)}{b_{i}\left(x^{5}\right)} t .
$$

One gets

$$
\left\{\begin{array}{l}
\left(x^{\prime}\right)_{(g), D S R 4}^{i}\left(\{x\}_{m .}, x^{5}\right)=\left(x^{i}\right)_{(g), D S R 4}^{\prime}\left(\{x\}_{m .}, x^{5}\right)=\widetilde{\gamma}(g)\left(x^{i}-\widetilde{\beta^{i}}(g) \tilde{x}^{0}\right) \\
\left(x^{\prime}\right)_{(g), D S R 4}^{k \neq i}\left(\{x\}_{m .}, x^{5}\right)=\left(x^{k \neq i}\right)_{(g), D S R 4}^{\prime}\left(\{x\}_{m .}, x^{5}\right)=x^{k \neq i} \\
\left(\tilde{x}^{0}\right)_{(g), D S R 4}^{\prime}\left(\{x\}_{m .}, x^{5}\right)=\widetilde{\gamma}(g)\left(\tilde{x}^{0}-\widetilde{\beta^{i}}(g) x^{i}\right) .
\end{array}\right.
$$

Such a symmetry is lost if we use the "standard" time coordinate $x^{0} \equiv c t$, which is related to $\tilde{x}^{0}$ by

$$
\tilde{x}^{0}=x^{0} \frac{b_{0}\left(x^{5}\right)}{b_{i}\left(x^{5}\right)} .
$$

In terms of $x^{0}$, we have in fact

$$
\left\{\begin{array}{l}
\left(x^{\prime}\right)_{(g), D S R 4}^{i}\left(\{x\}_{m .}, x^{5}\right)=\left(x^{i}\right)_{(g), D S R 4}^{\prime}\left(\{x\}_{m .}, x^{5}\right)=\widetilde{\gamma}(g)\left(x^{i}-\widetilde{\beta^{i}}(g) \frac{b_{0}\left(x^{5}\right)}{b_{i}\left(x^{5}\right)} x^{0}\right) \\
\left(x^{\prime}\right)_{(g), D S R 4}^{k \neq i}\left(\{x\}_{m .}, x^{5}\right)=\left(x^{k \neq i}\right)_{(g), D S R 4}^{\prime}\left(\{x\}_{m .}, x^{5}\right)=x^{k \neq i} \\
\left(x^{\prime}\right)_{(g), D S R 4}^{0}\left(\{x\}_{m .}, x^{5}\right)=\left(x^{0}\right)_{(g), D S R 4}^{\prime}\left(\{x\}_{m .}, x^{5}\right)=\widetilde{\gamma}(g)\left(x^{0}-\widetilde{\beta^{i}}(g) \frac{b_{i}\left(x^{5}\right)}{b_{0}\left(x^{5}\right)} x^{i}\right) .
\end{array}\right.
$$


Comparing Eq. (54) with Eq. (45) allows us to get the relations connecting the dimensional parametric basis of velocities $\left\{v^{i}\right\}$ (introduced in Ref. [10]) and the dimensionless basis of (effective) deformed rapidities $\left\{\widetilde{\zeta}^{i}\left(g, x^{5}\right)\right\}$ (defined by (44)) (the dependence on $x^{5}$ is now fully explicited):

$$
\forall i=1,2,3\left\{\begin{array}{l}
I) \quad \cosh \tilde{\zeta}_{i}\left(g, x^{5}\right)=\widetilde{\gamma}\left(g, x^{5}\right)= \\
=\left(1-\left(\widetilde{\beta^{i}}\left(g, x^{5}\right)\right)^{2}\right)^{-1 / 2}=\left(1-\left(\frac{v^{i}(g) b_{i}\left(x^{5}\right)}{c b_{0}\left(x^{5}\right)}\right)^{2}\right)^{-1 / 2} \\
=\frac{v^{i}(g) b_{i}^{2}\left(x^{5}\right)}{c b_{0}^{2}\left(x^{5}\right)}\left(1-\left(\frac{v^{i}(g) b_{i}\left(x^{5}\right)}{c b_{0}\left(x^{5}\right)}\right)^{2}\right)^{-1 / 2} \\
I I) b_{0}^{-1}\left(x^{5}\right) b_{i}\left(x^{5}\right)\left(\sinh \tilde{\zeta}_{i}\left(g, x^{5}\right)\right)=\widetilde{\gamma}\left(g, x^{5}\right) \widetilde{\beta^{i}}\left(g, x^{5}\right) \frac{b_{i}\left(x^{5}\right)}{b_{0}\left(x^{5}\right)}= \\
I I I) \quad b_{0}\left(x^{5}\right) b_{i}^{-1}\left(x^{5}\right)\left(\sinh \tilde{\zeta}_{i}\left(g, x^{5}\right)\right)=\widetilde{\gamma}\left(g, x^{5}\right) \widetilde{\beta^{i}}\left(g, x^{5}\right) \frac{b_{0}\left(x^{5}\right)}{b_{i}\left(x^{5}\right)}= \\
=\frac{v^{i}(g)}{c}\left(1-\left(\frac{v^{i}(g) b_{i}\left(x^{5}\right)}{c b_{0}\left(x^{5}\right)}\right)^{2}\right)^{-1 / 2}
\end{array}\right.
$$

From the above system one gets (ESC off):

$$
\left(1-\left(\frac{v^{i}(g) b_{i}\left(x^{5}\right)}{c b_{0}\left(x^{5}\right)}\right)^{2}\right)^{-1 / 2}=\widetilde{\gamma}\left(g, x^{5}\right) \equiv \widetilde{\gamma}^{i}\left(g, x^{5}\right)=\cosh \tilde{\zeta}_{i}\left(g, x^{5}\right)
$$




$$
\begin{gathered}
\frac{v^{i}(g) b_{i}\left(x^{5}\right)}{c b_{0}\left(x^{5}\right)}\left(1-\left(\frac{v^{i}(g) b_{i}\left(x^{5}\right)}{c b_{0}\left(x^{5}\right)}\right)^{2}\right)^{-1 / 2} \\
=\widetilde{\gamma}\left(g, x^{5}\right) \widetilde{\beta^{i}}\left(g, x^{5}\right) \equiv \widetilde{\gamma}^{i}\left(g, x^{5}\right) \widetilde{\beta^{i}}\left(g, x^{5}\right)= \\
=\sinh \tilde{\zeta}_{i}\left(g, x^{5}\right) .
\end{gathered}
$$

Such relations are consistent with the properties of hyperbolic functions, since $(\forall i=1,2,3)$ (ESC off)

$$
\begin{gathered}
\cosh ^{2} \tilde{\zeta}_{i}\left(g, x^{5}\right)-\sinh ^{2} \tilde{\zeta}_{i}\left(g, x^{5}\right)=1 \Leftrightarrow \\
\Leftrightarrow\left(1-\left(\frac{v^{i}(g) b_{i}\left(x^{5}\right)}{c b_{0}\left(x^{5}\right)}\right)^{2}\right)^{-1}-\left(\frac{v^{i}(g) b_{i}\left(x^{5}\right)}{c b_{0}\left(x^{5}\right)}\right)^{2}\left(1-\left(\frac{v^{i}(g) b_{i}\left(x^{5}\right)}{c b_{0}\left(x^{5}\right)}\right)^{2}\right)^{-1}= \\
=\frac{\left(1-\left(\frac{v^{i}(g) b_{i}\left(x^{5}\right)}{c b_{0}\left(x^{5}\right)}\right)^{2}\right)}{\left(1-\left(\frac{v^{i}(g) b_{i}\left(x^{5}\right)}{c b_{0}\left(x^{5}\right)}\right)^{2}\right)}=1
\end{gathered}
$$

Eqs. (55) and (56) reduce of course to the standard SR relations in the limit $\widetilde{M}_{4}\left(x^{5}\right) \longrightarrow M_{4}$.

\subsection{Finite Deformed Rotation About a Coordinate Axis}

Let us now consider a finite true (clockwise) deformed rotation by an angle $\theta_{1}(g)$ about $\widehat{x^{1}}$. By recalling that $\mathbf{S}_{D S R 4}\left(x^{5}\right) \equiv\left(I_{D S R 4}^{23}\left(x^{5}\right), I_{D S R 4}^{31}\left(x^{5}\right), I_{D S R 4}^{12}\left(x^{5}\right)\right)$, it follows: 


$$
\begin{aligned}
& S O(3,1)_{D E F .} \ni g: x^{\mu} \rightarrow x_{(g)}^{\mu \prime}\left(\{x\}_{m .}, x^{5}\right)= \\
& =\exp \left(-\theta_{1}(g) S_{D S R 4}^{1}\left(x^{5}\right)\right)^{\mu}{ }_{\nu} x^{\nu}=\exp \left(\theta_{1}(g) I_{D S R 4}^{32}\left(x^{5}\right)\right)^{\mu}{ }_{\nu} x^{\nu}= \\
& =\left(\sum_{n=0}^{\infty} \frac{1}{n !}\left(\theta_{1}(g) I_{D S R 4}^{32}\left(x^{5}\right)\right)^{n}\right)_{\nu}^{\mu} x^{\nu}=\left(\sum_{n=0}^{\infty} \frac{1}{n !}\left(\theta_{1}(g)\right)^{n}\left(I_{D S R 4}^{32}\left(x^{5}\right)\right)^{n}\right)_{\nu}^{\mu} x^{\nu}= \\
& =\left(1_{4-d .}+\sum_{n=1}^{\infty} \frac{1}{(2 n) !}\left(\theta_{1}(g)\right)^{2 n}\left(I_{D S R 4}^{32}\left(x^{5}\right)\right)^{2 n}+\right. \\
& \left.+\sum_{n=0}^{\infty} \frac{1}{(2 n+1) !}\left(\theta_{1}(g)\right)^{2 n+1}\left(I_{D S R 4}^{32}\left(x^{5}\right)\right)^{2 n+1}\right)_{\nu}^{\mu} x^{\nu}= \\
& =\left(1_{4-d .}+B_{1} \sum_{n=1}^{\infty} \frac{(-1)^{n}}{(2 n) !} b_{2}^{-2 n}\left(x^{5}\right) b_{3}^{-2 n}\left(x^{5}\right)\left(\theta_{1}(g)\right)^{2 n}+\right. \\
& \left.\left.+I_{D S R 4}^{32}\left(x^{5}\right) \sum_{n=0}^{\infty} \frac{(-1)^{n}}{(2 n+1) !} b_{2}^{-2 n}\left(x^{5}\right) b_{3}^{-2 n}\left(x^{5}\right)\left(\theta_{1}(g)\right)^{2 n+1}\right)\right)_{\nu}^{\mu} x^{\nu}= \\
& =\left(1_{4-d .}+B_{1} \sum_{n=1}^{\infty} \frac{(-1)^{n}}{(2 n) !}\left(\theta_{1}(g) b_{2}^{-1}\left(x^{5}\right) b_{3}^{-1}\left(x^{5}\right)\right)^{2 n}+\right. \\
& \left.+I_{D S R 4}^{32}\left(x^{5}\right) b_{2}\left(x^{5}\right) b_{3}\left(x^{5}\right) \sum_{n=0}^{\infty} \frac{(-1)^{n}}{(2 n+1) !}\left(\theta_{1}(g) b_{2}^{-1}\left(x^{5}\right) b_{3}^{-1}\left(x^{5}\right)\right)^{2 n+1}\right)_{\nu}^{\mu} x^{\nu}= \\
& =\left(1_{4-d .}+\left(\left(\cos \theta_{1}(g) b_{2}^{-1}\left(x^{5}\right) b_{3}^{-1}\left(x^{5}\right)\right)-1\right) B_{1}+\right. \\
& \left.+b_{2}\left(x^{5}\right) b_{3}\left(x^{5}\right)\left(\sin \theta_{1}(g) b_{2}^{-1}\left(x^{5}\right) b_{3}^{-1}\left(x^{5}\right)\right) I_{D S R 4}^{32}\left(x^{5}\right)\right)_{\nu}^{\mu} x^{\nu},
\end{aligned}
$$


where in the last passage the series expansions of trigonometric functions have been used.

By introducing the matrix

$$
\begin{gathered}
\text { Rot. }_{D S R 4, \widehat{x}^{1}}\left(g, x^{5}\right) \equiv 1_{4-d .}+\left(\left(\cos \theta_{1}(g) b_{2}^{-1}\left(x^{5}\right) b_{3}^{-1}\left(x^{5}\right)\right)-1\right) B_{1}+ \\
+b_{2}\left(x^{5}\right) b_{3}\left(x^{5}\right)\left(\sin \theta_{1}(g) b_{2}^{-1}\left(x^{5}\right) b_{3}^{-1}\left(x^{5}\right)\right) I_{D S R 4}^{32}\left(x^{5}\right),
\end{gathered}
$$

Eq. (59) can be rewritten as:

$$
\begin{gathered}
S O(3,1)_{D E F .} \ni g: x^{\mu} \rightarrow \\
=\left(x^{\prime}\right)_{(g), D S R 4}^{\mu}\left(\{x\}_{m .}, x^{5}\right)=\left(x^{\mu}\right)_{(g), D S R 4}^{\prime}\left(\{x\}_{m .}, x^{5}\right)= \\
=\left(\text { Rot. }_{D S R 4, \widehat{x^{1}}}\left(g, x^{5}\right)\right)_{\nu}^{\mu} x^{\nu} .
\end{gathered}
$$

We finally have:

$$
\begin{aligned}
& \left(\begin{array}{c}
\left(x^{\prime}\right)_{(g), D S R 4}^{0}\left(\{x\}_{m .}, x^{5}\right) \\
\left(x^{\prime}\right)_{(g), D S R 4}^{1}\left(\{x\}_{m .}, x^{5}\right) \\
\left(x^{\prime}\right)_{(g), D S R 4}^{2}\left(\{x\}_{m .}, x^{5}\right) \\
\left(x^{\prime}\right)_{(g), D S R 4}^{3}\left(\{x\}_{m .}, x^{5}\right)
\end{array}\right)=\left(\begin{array}{c}
\left(x^{0}\right)_{(g), D S R 4}^{\prime}\left(\{x\}_{m .}, x^{5}\right) \\
\left(x^{1}\right)_{(g), D S R 4}^{\prime}\left(\{x\}_{m .}, x^{5}\right) \\
\left(x^{2}\right)_{(g), D S R 4}^{\prime}\left(\{x\}_{m .}, x^{5}\right) \\
\left(x^{3}\right)_{(g), D S R 4}^{\prime}\left(\{x\}_{m .}, x^{5}\right)
\end{array}\right)= \\
& =\left(\text { Rot. }_{D S R 4, \widehat{x^{1}}}\left(g, x^{5}\right)\right) \times\left(\begin{array}{c}
x^{0} \\
x^{1} \\
x^{2} \\
x^{3}
\end{array}\right)= \\
& =\left(\begin{array}{c}
x^{0} \\
x^{1} \\
\left(\cos \theta_{1}(g) b_{2}^{-1}\left(x^{5}\right) b_{3}^{-1}\left(x^{5}\right)\right) x^{2}+b_{2}^{-1}\left(x^{5}\right) b_{3}\left(x^{5}\right)\left(\sin \theta_{1}(g) b_{2}^{-1}\left(x^{5}\right) b_{3}^{-1}\left(x^{5}\right)\right) x^{3} \\
-b_{2}\left(x^{5}\right) b_{3}^{-1}\left(x^{5}\right)\left(\sin \theta_{1}(g) b_{2}^{-1}\left(x^{5}\right) b_{3}^{-1}\left(x^{5}\right)\right) x^{2}+\left(\cos \theta_{1}(g) b_{2}^{-1}\left(x^{5}\right) b_{3}^{-1}\left(x^{5}\right)\right) x^{3}
\end{array}\right) .
\end{aligned}
$$


Analogous relations hold for the finite true (clockwise) deformed rotations about the other two spatial axes. One has

$$
\begin{aligned}
S O(3,1)_{D E F .} \ni g: x^{\mu} \rightarrow & \left(x^{\prime}\right)_{(g), D S R 4}^{\mu}\left(\{x\}_{m .}, x^{5}\right)=\left(x^{\mu}\right)_{(g), D S R 4}^{\prime}\left(\{x\}_{m .}, x^{5}\right)= \\
& =\left(\text { Rot. }_{D S R 4, \widehat{x^{2}}}\left(g, x^{5}\right)\right)_{\nu}^{\mu} x_{\nu}^{\nu} ;
\end{aligned}
$$

$S O(3,1)_{D E F .} \ni g: x^{\mu} \rightarrow\left(x^{\prime}\right)_{(g), D S R 4}^{\mu}\left(\{x\}_{m .}, x^{5}\right)=\left(x^{\mu}\right)_{(g), D S R 4}^{\prime}\left(\{x\}_{m .}, x^{5}\right)=$

$$
=\left(\operatorname{Rot}_{{ }_{D S R 4}, \widehat{x^{3}}}\left(g, x^{5}\right)\right)_{\nu}^{\mu} x^{\nu},
$$

where we defined

$$
\begin{aligned}
& \operatorname{Rot}_{{ }_{D S R 4, \widehat{x^{2}}}}\left(g, x^{5}\right) \equiv 1_{4-d .}+\left(\left(\cos \theta_{2}(g) b_{1}^{-1}\left(x^{5}\right) b_{3}^{-1}\left(x^{5}\right)\right)-1\right) B_{2}+ \\
& +b_{1}\left(x^{5}\right) b_{3}\left(x^{5}\right)\left(\sin \theta_{2}(g) b_{1}^{-1}\left(x^{5}\right) b_{3}^{-1}\left(x^{5}\right)\right) I_{D S R 4}^{13}\left(x^{5}\right) ; \\
& \operatorname{Rot}_{{ }_{D S R 4, \widehat{x^{3}}}}\left(g, x^{5}\right) \equiv 1_{4-d .}+\left(\left(\cos \theta_{3}(g) b_{1}^{-1}\left(x^{5}\right) b_{2}^{-1}\left(x^{5}\right)\right)-1\right) B_{3}+ \\
& \quad+b_{1}\left(x^{5}\right) b_{2}\left(x^{5}\right)\left(\sin \theta_{3}(g) b_{1}^{-1}\left(x^{5}\right) b_{2}^{-1}\left(x^{5}\right)\right) I_{D S R 4}^{21}\left(x^{5}\right) .
\end{aligned}
$$

By introducing the (effective) deformed angles $\tilde{\theta}(g)(i \neq j, i \neq k, j \neq k)^{6}$

\footnotetext{
${ }^{6}$ Definition $(67)$ of $\tilde{\theta}(g)$ does formally coincides with that of the isotopic angles introduced by Santilli [11].
} 


$$
\tilde{\theta}_{i}\left(g, x^{5}\right) \equiv \theta_{i}(g) b_{j}^{-1}\left(x^{5}\right) b_{k}^{-1}\left(x^{5}\right)=
$$

ESC off on $i, \underset{\text { ESC on on } j \text { and } k}{=} \frac{1}{2} \theta_{i}(g)\left|\epsilon_{i j k}\right| b_{j}^{-1}\left(x^{5}\right) b_{k}^{-1}\left(x^{5}\right), \forall i=1,2,3$,

Eqs. (60), (61) and (63)-(66) can be written in compact form respectively as

$$
\begin{aligned}
& \left(\begin{array}{c}
\left(x^{\prime}\right)_{(g), D S R 4}^{0}\left(\{x\}_{m .}, x^{5}\right) \\
\left(x^{\prime}\right)_{(g), D S R 4}^{1}\left(\{x\}_{m .}, x^{5}\right) \\
\left(x^{\prime}\right)_{(g), D S R 4}^{2}\left(\{x\}_{m .}, x^{5}\right) \\
\left(x^{\prime}\right)_{(g), D S R 4}^{3}\left(\{x\}_{m .}, x^{5}\right)
\end{array}\right)=\left(\begin{array}{c}
\left(x^{0}\right)_{(g), D S R 4}^{\prime}\left(\{x\}_{m .}, x^{5}\right) \\
\left(x^{1}\right)_{(g), D S R 4}^{\prime}\left(\{x\}_{m .}, x^{5}\right) \\
\left(x^{2}\right)_{(g), D S R 4}^{\prime}\left(\{x\}_{m .}, x^{5}\right) \\
\left(x^{3}\right)_{(g), D S R 4}^{\prime}\left(\{x\}_{m .}, x^{5}\right)
\end{array}\right)= \\
& =\left(\begin{array}{c}
x^{0} \\
x^{1} \\
\left(\cos \tilde{\theta}_{1}\left(g, x^{5}\right)\right) x^{2}+b_{2}^{-1}\left(x^{5}\right) b_{3}\left(x^{5}\right)\left(\sin \tilde{\theta}_{1}\left(g, x^{5}\right)\right) x^{3} \\
-b_{2}\left(x^{5}\right) b_{3}^{-1}\left(x^{5}\right)\left(\sin \tilde{\theta}_{1}\left(g, x^{5}\right)\right) x^{2}+\left(\cos \tilde{\theta}_{1}\left(g, x^{5}\right)\right) x^{3}
\end{array}\right) ;
\end{aligned}
$$




$$
\begin{aligned}
& \left(\begin{array}{c}
\left(x^{\prime}\right)_{(g), D S R 4}^{0}\left(\{x\}_{m .}, x^{5}\right) \\
\left(x^{\prime}\right)_{(g), D S R 4}^{1}\left(\{x\}_{m .}, x^{5}\right) \\
\left(x^{\prime}\right)_{(g), D S R 4}^{2}\left(\{x\}_{m .}, x^{5}\right) \\
\left(x^{\prime}\right)_{(g), D S R 4}^{3}\left(\{x\}_{m .}, x^{5}\right)
\end{array}\right)=\left(\begin{array}{c}
\left(x^{0}\right)_{(g), D S R 4}^{\prime}\left(\{x\}_{m .}, x^{5}\right) \\
\left(x^{1}\right)_{(g), D S R 4}^{\prime}\left(\{x\}_{m .}, x^{5}\right) \\
\left(x^{2}\right)_{(g), D S R 4}^{\prime}\left(\{x\}_{m .}, x^{5}\right) \\
\left(x^{3}\right)_{(g), D S R 4}^{\prime}\left(\{x\}_{m .}, x^{5}\right)
\end{array}\right)= \\
& =\left(\begin{array}{c}
x^{0} \\
x^{1} \\
\left(\cos \tilde{\theta}_{1}\left(g, x^{5}\right)\right) x^{2}+b_{2}^{-1}\left(x^{5}\right) b_{3}\left(x^{5}\right)\left(\sin \tilde{\theta}_{1}\left(g, x^{5}\right)\right) x^{3} \\
-b_{2}\left(x^{5}\right) b_{3}^{-1}\left(x^{5}\right)\left(\sin \tilde{\theta}_{1}\left(g, x^{5}\right)\right) x^{2}+\left(\cos \tilde{\theta}_{1}\left(g, x^{5}\right)\right) x^{3}
\end{array}\right) \\
& \left(\begin{array}{c}
\left(x^{\prime}\right)_{(g), D S R 4}^{0}\left(\{x\}_{m .}, x^{5}\right) \\
\left(x^{\prime}\right)_{(g), D S R 4}^{1}\left(\{x\}_{m .}, x^{5}\right) \\
\left(x^{\prime}\right)_{(g), D S R 4}^{2}\left(\{x\}_{m .}, x^{5}\right) \\
\left(x^{\prime}\right)_{(g), D S R 4}^{3}\left(\{x\}_{m .}, x^{5}\right)
\end{array}\right)=\left(\begin{array}{c}
\left(x^{0}\right)_{(g), D S R 4}^{\prime}\left(\{x\}_{m .}, x^{5}\right) \\
\left(x^{1}\right)_{(g), D S R 4}^{\prime}\left(\{x\}_{m .}, x^{5}\right) \\
\left(x^{2}\right)_{(g), D S R 4}^{\prime}\left(\{x\}_{m .}, x^{5}\right) \\
\left(x^{3}\right)_{(g), D S R 4}^{\prime}\left(\{x\}_{m .}, x^{5}\right)
\end{array}\right)= \\
& =\left(\begin{array}{c}
x^{0} \\
\left(\cos \tilde{\theta}_{3}\left(g, x^{5}\right)\right) x^{1}+b_{1}^{-1}\left(x^{5}\right) b_{2}\left(x^{5}\right)\left(\sin \tilde{\theta}_{3}\left(g, x^{5}\right)\right) x^{2} \\
-b_{1}\left(x^{5}\right) b_{2}^{-1}\left(x^{5}\right)\left(\sin \tilde{\theta}_{3}\left(g, x^{5}\right)\right) x^{1}+\left(\cos \tilde{\theta}_{3}\left(g, x^{5}\right)\right) x^{2} \\
x^{3}
\end{array}\right) .
\end{aligned}
$$

By comparing the finite true (clockwise) rotations by an angle $\theta^{i}(g)$ about 
$\widehat{x^{i}}$ in SR

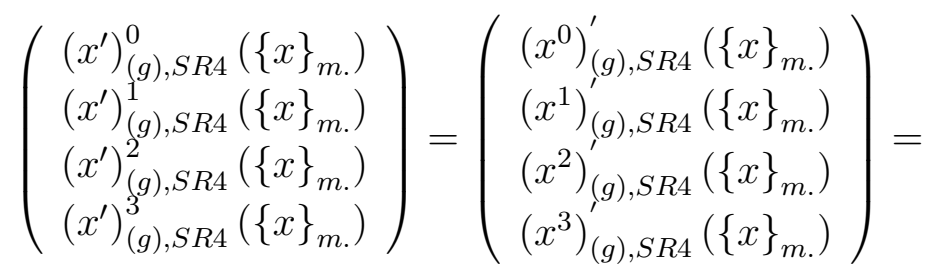

$$
\begin{aligned}
& =\left(\begin{array}{c}
x^{0} \\
x^{1} \\
\left(\cos \theta_{1}(g)\right) x^{2}+\left(\sin \theta_{1}(g)\right) x^{3} \\
-\left(\sin \theta_{1}(g)\right) x^{2}+\left(\cos \theta_{1}(g)\right) x^{3}
\end{array}\right) ;
\end{aligned}
$$

$$
\begin{gathered}
\left(\begin{array}{c}
\left(x^{\prime}\right)_{(g), S R 4}^{0}\left(\{x\}_{m .}\right) \\
\left(x^{\prime}\right)_{(g,), S 4}^{g}\left(\{x\}_{m .}\right) \\
\left(x^{\prime}\right)_{(g), S R}^{2}\left(\{x\}_{m .}\right) \\
\left(x^{\prime}\right)_{(g), S R 4}^{g}\left(\{x\}_{m .}\right)
\end{array}\right)=\left(\begin{array}{c}
\left(x^{0}\right)_{(g), S R 4}^{\prime}\left(\{x\}_{m}\right) \\
\left(x^{1}\right)_{(g), S R 4}\left(\{x\}_{m .}\right) \\
\left(x^{2}\right)_{(g), S R 4}^{\prime}\left(\{x\}_{m .}\right) \\
\left(x^{3}\right)_{(g), S R 4}\left(\{x\}_{m .}\right)
\end{array}\right)= \\
=\left(\begin{array}{c}
x^{0} \\
\left(\cos \theta_{2}(g)\right) x^{1}-\left(\sin \theta_{2}(g)\right) x^{3} \\
x^{2} \\
\left(\sin \theta_{2}(g)\right) x^{1}+\left(\cos \theta_{2}(g)\right) x^{3}
\end{array}\right) ;
\end{gathered}
$$




$$
\begin{gathered}
\left(\begin{array}{c}
\left(x^{\prime}\right)_{(g), S R 4}^{0}\left(\{x\}_{m .}\right) \\
\left(x^{\prime}\right)_{(g), S R 4}^{1}\left(\{x\}_{m .}\right) \\
\left(x^{\prime}\right)_{(g), S R 4}^{2}\left(\{x\}_{m .}\right) \\
\left(x^{\prime}\right)_{(g), S R 4}^{g}\left(\{x\}_{m .}\right)
\end{array}\right)=\left(\begin{array}{c}
\left(x^{0}\right)_{(g), S R 4}^{\prime}\left(\{x\}_{m .}\right) \\
\left(x^{1}\right)_{(g), S R 4}^{\prime}\left(\{x\}_{m .}\right) \\
\left(x^{2}\right)_{(g), S R 4}^{\prime}\left(\{x\}_{m .}\right) \\
\left(x^{3}\right)_{(g), S R 4}^{\prime}\left(\{x\}_{m .}\right)
\end{array}\right)= \\
=\left(\begin{array}{c}
\left(\cos \theta_{3}(g)\right) x^{1}+\left(\sin \theta_{3}(g)\right) x^{2} \\
-\left(\sin \theta_{3}(g)\right) x^{1}+\left(\cos \theta_{3}(g)\right) x^{2} \\
x^{3}
\end{array}\right)
\end{gathered}
$$

with those in DSR4, it is easily seen that the deforming transition SR $\rightarrow$ DSR4 corresponds - at the level of group parameters - to the deforming and anisotropizing rescaling of angles $\theta_{i}(g) \rightarrow \widetilde{\theta}_{i}\left(g, x^{5}\right), \forall i=1,2,3$.

Let us notice that the chronotopical rotation group $S O(3,1)_{G E N}$. of the generalized 4-d. ((3,1) hyperbolically-signed) Minkowski space is non-compact (like the standard one $S O(3,1)$ of usual SR). This is obviously related to the existence of at least one timelike dimension, namely of pseudo-rotations (or boost transformations). Indeed, whereas the range of the 3-vector angle parameter $\theta(g)$ is compact $\left(\theta^{i}(g) \in[0,2 \pi] \quad \forall i=1,2,3\right)$, each component of the rapidity 3 -vector $\zeta(g)$ has a non-compact range (the whole real line: $\left.\zeta^{i}(g) \in R \equiv(-\infty,+\infty) \quad \forall i=1,2,3\right)^{7}$.

${ }^{7}$ The same holds true of course for the velocity parameter. In SR, the light speed $c$ being the maximal causal velocity (m.c.v.) implies that the range of the dimensional boost parameter $v^{i}(g)$ is the real, non-compact (since bounded but open) interval $(-c,+c)$ $\forall g \in S O(3,1)_{S T D}$. (namely, "luminal" boosts are not allowed, what amounts to say that no rest frame exists for a massless particle.). Analogously, in DSR4, where the m.c.v. is given by $u_{D S R 4}^{i}=\frac{b_{0}\left(x^{5}\right)}{b_{i}\left(x^{5}\right)} c$, the range of the dimensional velocity parameter $v^{i}(g)$ of the deformed boost is the real, non-compact interval $\left(-u_{D S R 4}^{i}=-c \frac{b_{0}\left(x^{5}\right)}{b_{i}\left(x^{5}\right)},+u_{D S R 4}^{i}=+c \frac{b_{0}\left(x^{5}\right)}{b_{i}\left(x^{5}\right)}\right)$ :

$$
v^{i}(g) \in\left(-u_{D S R 4}^{i},+u_{D S R 4}^{i}\right), \forall i=1,2,3, \forall g \in S O(3,1)_{D E F .},
$$

since

$$
\left.\widetilde{\beta}^{i}(g)\right|_{v^{i}(g)= \pm u_{D S R 4}^{i}}= \pm \frac{u_{D S R 4}^{i}}{u_{D S R 4}^{i}}= \pm 1
$$


Such a conclusion holds true, in general, for the $N$-dimensional case. The presence of timelike dimensions (i.e. the fact $T>0$ ), and therefore of true space-time mixing, implies the lack of compactness of the chronotopical rotation group of the $N$-d. generalized Minkowski space, i.e. of the homogeneous component of the corresponding (maximal) Killing group $P(S, T)_{G E N \text {. }}$

\subsection{The Antisymmetric Tensor $\delta \omega_{D S R 4}^{\mu \nu}\left(g, x^{5}\right)$ of the Di- mensionless (Effective) Deformed Parameters of the Space-Time Rotation Group in DSR4}

It was shown in Ref. [1] that, for a generalized Minkowski space, all forms of the tensor $\delta \omega$ are global, i.e. independent of the set of metric variables

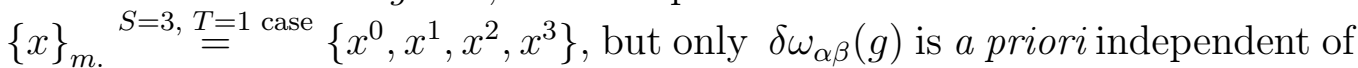
possible non-metric variables $\{x\}_{n . m}$. For instance, consider the completely contravariant form of $\delta \omega$ :

$$
\delta \omega^{\alpha \beta}\left(g,\{x\}_{n . m .}\right) \equiv g^{\alpha \gamma}\left(\{x\}_{n . m .}\right) g^{\beta \delta}\left(\{x\}_{n . m .}\right) \delta \omega_{\alpha \beta}(g)
$$

or, in matrix form:

$$
\delta \omega_{\text {contrav. }}\left(g,\{x\}_{n . m .}\right) \equiv g_{\text {contrav. }}^{T}\left(\{x\}_{n . m .}\right) \times \delta \omega_{\text {cov. }}(g) \times g_{\text {contrav. }}\left(\{x\}_{n . m .}\right) .
$$

In the DSR4 case, the completely contravariant metric tensor reads

$$
\begin{gathered}
g_{D S R 4}^{\mu \nu}\left(x^{5}\right)=\operatorname{diag}\left(b_{0}^{-2}\left(x^{5}\right),-b_{1}^{-2}\left(x^{5}\right),-b_{2}^{-2}\left(x^{5}\right),-b_{3}^{-2}\left(x^{5}\right)\right)= \\
\stackrel{E_{\text {Coff }}}{=} \delta^{\mu \nu}\left(\delta^{\mu 0} b_{0}^{-2}\left(x^{5}\right)-\delta^{\mu 1} b_{1}^{-2}\left(x^{5}\right)-\delta^{\mu 2} b_{2}^{-2}\left(x^{5}\right)-\delta^{\mu 3} b_{3}^{-2}\left(x^{5}\right)\right)
\end{gathered}
$$

$$
\begin{aligned}
\left.\tilde{\gamma^{i}}(g)\right|_{v^{i}(g)= \pm u_{D S R 4}^{i}} & =\left.\left[\left(1-\left(\widetilde{\beta^{i}}(g)\right)^{2}\right)^{-1 / 2}\right]\right|_{v^{i}(g)= \pm u_{D S R 4}^{i}}= \\
& =1-\left[\left(\left.\widetilde{\beta}^{i}(g)\right|_{v^{i}(g)= \pm u_{D S R 4}^{i}}\right)^{2}\right]^{-1 / 2}=\infty
\end{aligned}
$$


or

$$
g_{\text {contrav.DSR4 }}\left(x^{5}\right)=\left(\begin{array}{cccc}
b_{0}^{-2}\left(x^{5}\right) & 0 & 0 & 0 \\
0 & -b_{1}^{-2}\left(x^{5}\right) & 0 & 0 \\
0 & 0 & -b_{2}^{-2}\left(x^{5}\right) & 0 \\
0 & 0 & 0 & -b_{3}^{-2}\left(x^{5}\right)
\end{array}\right) \text {. }
$$


Therefore

$$
\begin{aligned}
& \delta \omega_{D S R 4}^{\alpha \beta}\left(g, x^{5}\right) \equiv \delta \omega_{\text {contrav.,DSR4 }}\left(g, x^{5}\right) \equiv \\
& \equiv g_{\text {contrav.DSR } 4}^{T}\left(x^{5}\right) \times \delta \omega_{\text {cov. },(D S R 4)}(g) \times g_{\text {contrav.DSR } 4}\left(x^{5}\right)= \\
& =\left(\begin{array}{cccc}
b_{0}^{-2}\left(x^{5}\right) & 0 & 0 & 0 \\
0 & -b_{1}^{-2}\left(x^{5}\right) & 0 & 0 \\
0 & 0 & -b_{2}^{-2}\left(x^{5}\right) & 0 \\
0 & 0 & 0 & -b_{3}^{-2}\left(x^{5}\right)
\end{array}\right) \times \\
& \times\left(\begin{array}{cccc}
0 & -\zeta^{1}(g) & -\zeta^{2}(g) & -\zeta^{3}(g) \\
\zeta^{1}(g) & 0 & -\theta^{3}(g) & \theta^{2}(g) \\
\zeta^{2}(g) & \theta^{3}(g) & 0 & -\theta^{1}(g) \\
\zeta^{3}(g) & -\theta^{2}(g) & \theta^{1}(g) & 0
\end{array}\right) \times \\
& \times\left(\begin{array}{cccc}
b_{0}^{-2}\left(x^{5}\right) & 0 & 0 & 0 \\
0 & -b_{1}^{-2}\left(x^{5}\right) & 0 & 0 \\
0 & 0 & -b_{2}^{-2}\left(x^{5}\right) & 0 \\
0 & 0 & 0 & -b_{3}^{-2}\left(x^{5}\right)
\end{array}\right)= \\
& =\left(\begin{array}{cccc}
b_{0}^{-2}\left(x^{5}\right) & 0 & 0 & 0 \\
0 & -b_{1}^{-2}\left(x^{5}\right) & 0 & 0 \\
0 & 0 & -b_{2}^{-2}\left(x^{5}\right) & 0 \\
0 & 0 & 0 & -b_{3}^{-2}\left(x^{5}\right)
\end{array}\right) \times \\
& \times\left(\begin{array}{cccc}
0 & \zeta^{1}(g) b_{1}^{-2}\left(x^{5}\right) & \zeta^{2}(g) b_{2}^{-2}\left(x^{5}\right) & \zeta^{3}(g) b_{3}^{-2}\left(x^{5}\right) \\
\zeta^{1}(g) b_{0}^{-2}\left(x^{5}\right) & 0 & \theta^{3}(g) b_{2}^{-2}\left(x^{5}\right) & -\theta^{2}(g) b_{3}^{-2}\left(x^{5}\right) \\
\zeta^{2}(g) b_{0}^{-2}\left(x^{5}\right) & -\theta^{3}(g) b_{1}^{-2}\left(x^{5}\right) & 0 & \theta^{1}(g) b_{3}^{-2}\left(x^{5}\right) \\
\zeta^{3}(g) b_{0}^{-2}\left(x^{5}\right) & \theta^{2}(g) b_{1}^{-2}\left(x^{5}\right) & -\theta^{1}(g) b_{2}^{-2}\left(x^{5}\right) & 0
\end{array}\right)
\end{aligned}
$$


whence, by recalling the definitions (44) and (67) of the (effective) deformed rapidity and angle (Euclidean) 3-vectors, we obtain :

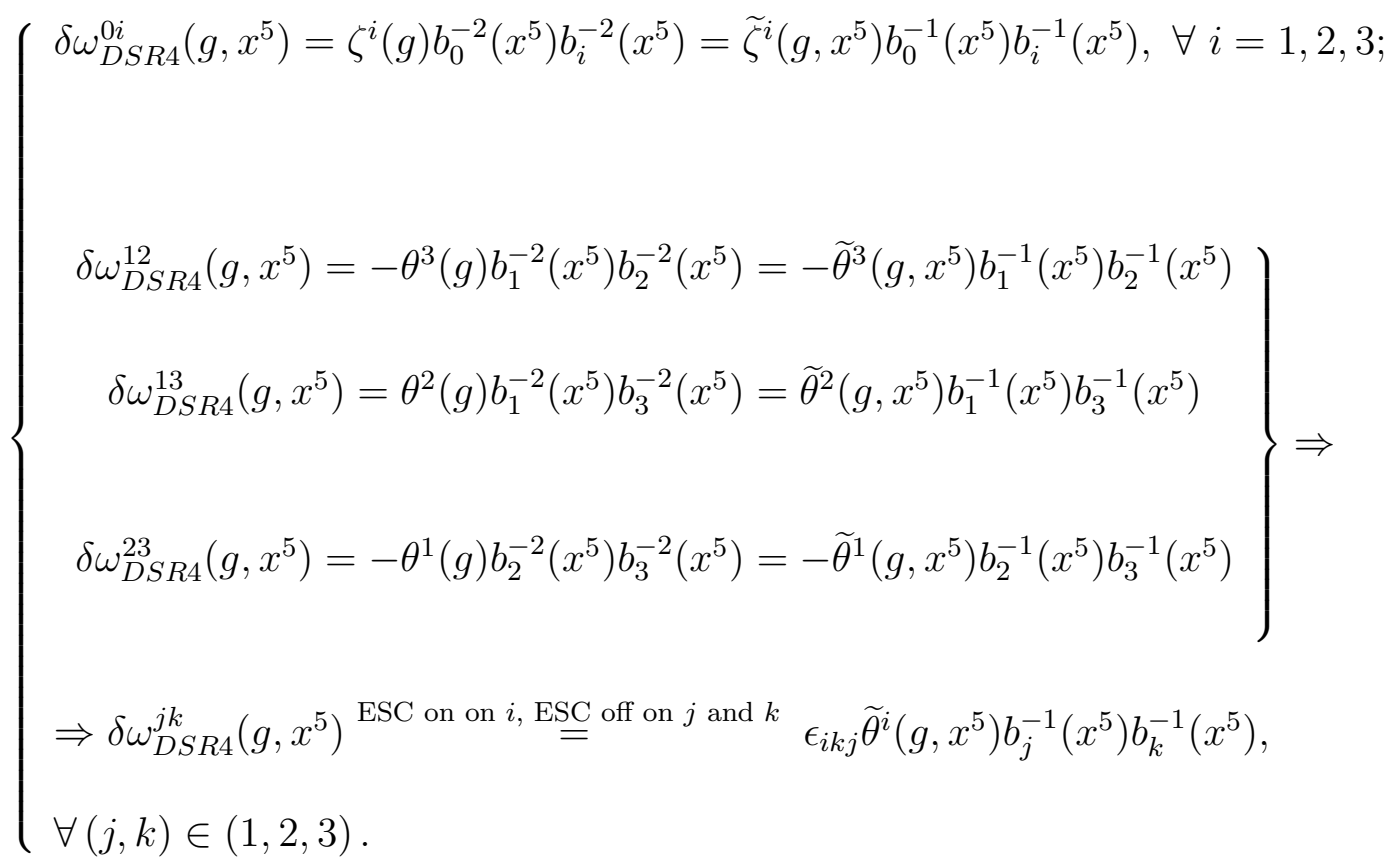

The above relation between the components of $\delta \omega_{\alpha \beta,(D S R 4)}(g)$ and those of $\delta \omega_{D S R 4}^{\alpha \beta}\left(g, x^{5}\right)$ can be therefore written as $(i, j=1,2,3)$ (ESC off):

$$
\begin{gathered}
\delta \omega_{i j,(D S R 4)}(g)=b_{i}^{2}\left(x^{5}\right) b_{j}^{2}\left(x^{5}\right) \delta \omega_{D S R 4}^{i j}\left(g, x^{5}\right) ; \\
\delta \omega_{0 i,(D S R 4)}(g)=-b_{0}^{-2}\left(x^{5}\right) b_{i}^{-2}\left(x^{5}\right) \delta \omega_{D S R 4}^{0 i}\left(g, x^{5}\right) .
\end{gathered}
$$

We can therefore state that the formal "anisotropizing deforming" transition $\mathrm{SR} \rightarrow \mathrm{DSR} 4$ is summarized, at the group parameter level, by the passage from the antisymmetric, parametric covariant tensor $\delta \omega_{\alpha \beta,(S S R 4)}(g)^{8}$ to the

${ }^{8}$ Notice that formally

$$
\delta \omega_{\alpha \beta,(S S R 4)}(g)=\delta \omega_{\alpha \beta,(D S R 4)}(g),
$$

but the $g$ 's belong to different space-time rotation groups. In the l.h.s of (०) $g \in$ $S O(3,1)_{S T D}$. (homogeneous Lorentz group), while in the r.h.s. of (o) $g$ belong to the "deformed" counterpart, i.e. $g \in S O(3,1)_{D E F}$. (homogeneous "deformed" Lorentz group). 
antisymmetric contravariant tensor $\delta \omega_{D S R 4}^{\alpha \beta}\left(g, x^{5}\right)$ of the (effective) deformed parameters of $S O(3,1)_{D E F .}{ }^{9}$.

\footnotetext{
${ }^{9}$ Let us notice that the same conclusion (namely, the characterization of $\delta \omega_{D S R 4}^{\alpha \beta}\left(g, x^{5}\right)$ as tensor of the effective deformed parameters) does not hold at the infinitesimal level. Indeed, in DSR4 (see Ref. [1]) — and in SR as well — one cannot rescale parameters in a unique way, since in any fixed infinitesimal transformation tipology the related group parameter is rescaled in a different way, depending on the coordinate concerned. Effective deformed group parameters can be therefore defined only at the finite level of the spacetime rotation component $S O(3,1)_{D E F}$. of the maximal Killing group $P(3,1)_{D E F}$. of the deformed 4-d. Minkowski space $\widetilde{M}_{4}\left(x^{5}\right)$.

The possibility of generalizing such results to the generalized 4-d. $(S, T=4-S)$ (and possibly to the $N$-d. $(S, T=N-S)$ ) Minkowskian spaces depends strongly on the explicit form of the metric tensor. For instance, for a non-diagonal $N$-d. metric tensor, the parametric tensor $\delta \omega^{\alpha \beta}\left(g,\{x\}_{n . m .}\right)$ in general has no definite symmetry property, and therefore it is more difficult (or even impossible) to identify its components with possible (effective) generalized parameters.
} 


\section{FINITE 3-d. DEFORMED TRUE ROTA- TIONS ABOUT A GENERIC AXIS IN DSR4}

\subsection{Parametric Decomposition}

We want now to derive the finite, deformed true (clockwise) rotations by a generic angle $\varphi(g)$ about a generic axis $\widehat{\varepsilon}(g)$ in the physical 3-d. space $\widetilde{E}_{3}\left(x^{5}\right)$ embedded in $M_{4}\left(x^{5}\right)$, by exploiting the form of the infinitesimal generators of the DSR4 chronotopical group $S O(3,1)_{D E F}$. obtained in Ref. [1]. The metric structure in $\widetilde{E_{3}}\left(x^{5}\right)$ is determined by $-g_{i j, D S R 4}\left(x^{5}\right) \stackrel{\mathrm{ESC}}{=}{ }^{\mathrm{Eff}} \delta_{i j} b_{i}^{2}\left(x^{5}\right)^{10}$. The unit vector of the rotation axis is

$$
\widehat{\varepsilon}(g)=\varepsilon^{1}(g) \widehat{x^{1}}+\varepsilon^{2}(g) \widehat{x^{2}}+\varepsilon^{3}(g) \widehat{x^{3}},
$$

with

$$
|\widehat{\varepsilon}(g)|_{*}^{2} \equiv \sum_{i=1}^{3} b_{i}^{2}\left(x^{5}\right)\left(\varepsilon^{i}(g)\right)^{2}=1
$$

(the $|\cdot|_{*}$ is the 3 -d. norm associated to $\left.-g_{i j, D S R 4}\left(x^{5}\right)\right)$.

In general, by the very basic properties of a group, it is always possible to find a (not unique) axial-parametric decomposition which transfers - once fixed the three coordinate axes in the space considered - all the dependence on the group element $g$ only on the transformation parameters $\left\{\theta^{i}(g)\right\}_{i=1,2,3}$

$$
(\widehat{\varepsilon}(g), \varphi(g)) \rightarrow\left(\widehat{x^{1}}, \theta^{1}(g)\right)\left(\widehat{x^{2}}, \theta^{2}(g)\right)\left(\widehat{x^{3}}, \theta^{3}(g)\right) .
$$

At the infinitesimal (i.e. algebraic) level, such a decomposition is independent on the order, due to the commutativity of the infinitesimal elements (i.e. of the transformations at an algebraic level) of any Lie group of transformations. This of course does not longer hold at a finite (i.e. group) level - due to the non-Abelian nature of Lie groups of chronotopical transformations - and therefore the order on the rhs of Eq. (81) is fixed for a given pair $(\widehat{\varepsilon}(g), \varphi(g))$ 11 .

\footnotetext{
${ }^{10}$ The minus sign is introduced to obtain positive 3-vector norms $\forall x^{5} \in R_{0}^{+}$.

${ }^{11}$ Needless to say, at the algebraic and group level rotation angles $\varphi(g)$ and $\left\{\theta^{i}(g)\right\}_{i=1,2,3}$ are infinitesimal and finite, respectively. This will be understood, and, for simplicity's sake, no notational distinction will be made.
} 
We have therefore, in DSR4 (· is the Euclidean scalar product):

$$
\begin{gathered}
-\varphi(g) \hat{\varepsilon}(g) \cdot \mathbf{S}_{D S R 4}\left(x^{5}\right) \rightarrow \\
\rightarrow-\theta^{1}(g) S_{D S R 4}^{1}\left(x^{5}\right)-\theta^{2}(g) S_{D S R 4}^{2}\left(x^{5}\right)-\theta^{3}(g) S_{D S R 4}^{3}\left(x^{5}\right)= \\
=-\sum_{i=1}^{3} \theta^{i}(g) S_{D S R 4}^{i}\left(x^{5}\right)
\end{gathered}
$$

(infinitesimal level, any composition order) ${ }^{12}$;

$$
\begin{gathered}
\exp \left(-\varphi(g) \widehat{\varepsilon}(g) \cdot \mathbf{S}_{D S R 4}\left(x^{5}\right)\right) \rightarrow \\
\rightarrow \exp \left(-\theta^{1}(g) S_{D S R 4}^{1}\left(x^{5}\right)\right) \times \exp \left(-\theta^{2}(g) S_{D S R 4}^{2}\left(x^{5}\right)\right) \times \exp \left(-\theta^{3}(g) S_{D S R 4}^{3}\left(x^{5}\right)\right)
\end{gathered}
$$

(finite level, fixed composition order).

A different axial-parametric decomposition utilizes the Euler angles $\left\{\theta_{1}^{\bar{i}}(g), \theta^{\bar{j} \neq \bar{i}}(g), \theta_{2}^{\bar{i}}(g)\right\}$ :

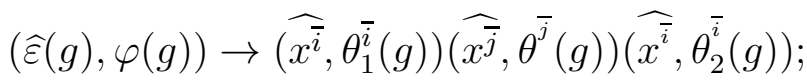

$$
\begin{aligned}
& \bar{i}, \bar{j} \in\{1,2,3\}, \bar{i} \neq \bar{j}
\end{aligned}
$$

In such a case, only two coordinate space axes, $\widehat{x^{\bar{i}}}$ and $\widehat{x^{\bar{j} \neq \bar{i}}}$, are used, with a special composition order. At infinitesimal level, one has

$$
\begin{aligned}
& (\widehat{\varepsilon}(g), \varphi(g)) \rightarrow \\
& \left.\left.\left.\left.\rightarrow \widehat{\left(x^{\bar{i}}\right.}, \theta_{1}^{\bar{i}}(g)\right)\left(\widehat{x^{\bar{j} \neq \bar{i}}}, \theta^{\bar{j} \neq \bar{i}}(g)\right) \widehat{x^{\bar{i}}}, \theta_{2}^{\bar{i}}(g)\right) \equiv \widehat{x^{\bar{i}}}, \theta_{1}^{\bar{i}}(g)+\theta_{2}^{\bar{i}}(g)\right) \widehat{x^{\bar{i}}}, \theta_{2}^{\bar{i}}(g)\right) \text {. }
\end{aligned}
$$

\footnotetext{
${ }^{12}$ To be more precise, in Eq. (82) - and in general for infinitesimal, deformed true rotation transformations - $g \in S O(3)_{D E F}$. should be replaced by $\delta g$, where $\delta g \in s u(2)_{D E F}$., i.e. it is an element of the deformed true rotation algebra. But, for simplicity's sake, we will omit, but mean, this cumbersome notation.
} 
For DSR4, we have

$$
\begin{gathered}
-\varphi(g) \widehat{\varepsilon}(g) \cdot \mathbf{S}_{D S R 4}\left(x^{5}\right) \rightarrow-\theta_{1}^{\bar{i}}(g) S_{D S R 4}^{\bar{i}}\left(x^{5}\right)-\theta^{\bar{j} \neq \bar{i}}(g) S_{D S R 4}^{\bar{j} \neq \bar{i}}\left(x^{5}\right)-\theta_{2}^{\bar{i}}(g) S_{D S R 4}^{\bar{i}}\left(x^{5}\right)= \\
=-\left(\theta_{1}^{\bar{i}}(g)+\theta_{2}^{\bar{i}}(g)\right) S_{D S R 4}^{\bar{i}}\left(x^{5}\right)-\theta^{\bar{j} \neq \bar{i}}(g) S_{D S R 4}^{\bar{j} \neq \bar{i}}\left(x^{5}\right)
\end{gathered}
$$

(infinitesimal level, any composition order);

$$
\begin{gathered}
\exp \left(-\varphi(g) \widehat{\varepsilon}(g) \cdot \mathbf{S}_{D S R 4}\left(x^{5}\right)\right) \rightarrow \\
\rightarrow \exp \left(-\theta_{1}^{\bar{i}}(g) S_{D S R 4}^{\bar{i}}\left(x^{5}\right)\right) \times \exp \left(-\theta^{\bar{j} \neq \bar{i}}(g) S_{D S R 4}^{\bar{j} \neq \bar{i}}\left(x^{5}\right)\right) \times \exp \left(-\theta_{2}^{\bar{i}}(g) S_{D S R 4}^{\bar{i}}\left(x^{5}\right)\right)
\end{gathered}
$$

(finite level, fixed composition order).

It is also possible to find out by direct computation (namely, by integration on the group parameters) the $4 \times 4$ matrix representative of the finite element $g$ of the rotation group $S O(3,1)_{D E F}$. in DSR4 corresponding to a rotation by $\varphi(g)$ about $\widehat{\varepsilon}(g)$. We have

$$
\begin{gathered}
-\varphi(g) \widehat{\varepsilon}(g) \cdot \mathbf{S}_{D S R 4}\left(x^{5}\right)=-\varphi(g) \sum_{i=1}^{3} \varepsilon^{i}(g) S_{D S R 4}^{i}\left(x^{5}\right) \rightarrow \\
\rightarrow \exp \left(-\varphi(g) \widehat{\varepsilon}(g) \cdot \mathbf{S}_{D S R 4}\left(x^{5}\right)\right)=\exp \left(-\varphi(g) \sum_{i=1}^{3} \varepsilon^{i}(g) S_{D S R 4}^{i}\left(x^{5}\right)\right) \neq \\
\neq \prod_{i=1}^{3} \exp \left(-\varphi(g) \varepsilon^{i}(g) S_{D S R 4}^{i}\left(x^{5}\right)\right)
\end{gathered}
$$

where in the last passage the Baker-Campbell-Hausdorff formula [12] is exploited, the non-Abelian nature of $S O(3)_{D E F} \subset S O(3,1)_{D E F}$. is used, and matrix product is understood in $\prod_{i=1}^{3}$.

Needless to say, all the above procedures are equivalent (although they may yield different formal results). Let us exploit the first one. 


\subsection{Exponentiating the Deformed Infinitesimal Rota- tion}

Let us denote by $A_{(\varphi(g), \widehat{\varepsilon}(g)), D S R 4}\left(x^{5}\right)$ the $4 \times 4$ matrix corresponding to an infinitesimal (clockwise) rotation by an (infinitesimal) angle $\varphi(g)$ about the axis $\widehat{\varepsilon}(g)$ (matrix belonging a 4-d. representation of the algebra of $S O(3)_{D E F}$, namely $\left.s u(2)_{D E F}\right)$ :

$$
\begin{gathered}
A_{(\varphi(g), \widehat{\varepsilon}(g)), D S R 4}\left(x^{5}\right) \equiv-\varphi(g) \widehat{\varepsilon}(g) \cdot \mathbf{S}_{D S R 4}\left(x^{5}\right) \rightarrow \\
\rightarrow\left(-\theta^{1}(g) S_{D S R 4}^{1}\left(x^{5}\right)-\theta^{2}(g) S_{D S R 4}^{2}\left(x^{5}\right)-\theta^{3}(g) S_{D S R 4}^{3}\left(x^{5}\right)\right) \rightarrow \\
\rightarrow \exp \left(A_{(\varphi(g), \widehat{\varepsilon}(g)), D S R 4}\left(x^{5}\right)\right)= \\
=\exp \left(-\theta^{1}(g) S_{D S R 4}^{1}\left(x^{5}\right)-\theta^{2}(g) S_{D S R 4}^{2}\left(x^{5}\right)-\theta^{3}(g) S_{D S R 4}^{3}\left(x^{5}\right)\right)= \\
=\sum_{n=0}^{\infty} \frac{1}{n !}\left(-\theta^{1}(g) S_{D S R 4}^{1}\left(x^{5}\right)-\theta^{2}(g) S_{D S R 4}^{2}\left(x^{5}\right)-\theta^{3}(g) S_{D S R 4}^{3}\left(x^{5}\right)\right)^{n},
\end{gathered}
$$

where $\exp \left(A_{(\varphi(g), \widehat{\varepsilon}(g)), D S R 4}\left(x^{5}\right)\right)$ is the $4 \times 4$ matrix corresponding to a finite (clockwise) rotation by a (finite) angle $\varphi(g)$ about the axis $\widehat{\varepsilon}(g)$, belonging to a 4-d. representation of $S O(3)_{D E F}$, of course.

On account of the explicit form of $\mathbf{S}_{D S R 4}\left(x^{5}\right)$ and of the deformed rotation generators (see Subsect. 4.1), we have

$$
\begin{gathered}
A_{(\varphi(g), \widehat{\varepsilon}(g)), D S R 4}\left(x^{5}\right)= \\
=\left(\begin{array}{cccc}
0 & 0 & 0 & 0 \\
0 & 0 & \theta^{3}(g) b_{1}^{-2}\left(x^{5}\right) & -\theta^{2}(g) b_{1}^{-2}\left(x^{5}\right) \\
0 & -\theta^{3}(g) b_{2}^{-2}\left(x^{5}\right) & 0 & \theta^{1}(g) b_{2}^{-2}\left(x^{5}\right) \\
0 & \theta^{2}(g) b_{3}^{-2}\left(x^{5}\right) & -\theta^{1}(g) b_{3}^{-2}\left(x^{5}\right) & 0
\end{array}\right),
\end{gathered}
$$


and therefore the corresponding finite form is given by the matrix exponential

$$
\begin{gathered}
\exp \left(A_{(\varphi(g), \widehat{\varepsilon}(g)), D S R 4}\left(x^{5}\right)\right)= \\
=\exp \left(\begin{array}{cccc}
0 & 0 & 0 & 0 \\
0 & 0 & \theta^{3}(g) b_{1}^{-2}\left(x^{5}\right) & -\theta^{2}(g) b_{1}^{-2}\left(x^{5}\right) \\
0 & -\theta^{3}(g) b_{2}^{-2}\left(x^{5}\right) & 0 & \theta^{1}(g) b_{2}^{-2}\left(x^{5}\right) \\
0 & \theta^{2}(g) b_{3}^{-2}\left(x^{5}\right) & -\theta^{1}(g) b_{3}^{-2}\left(x^{5}\right) & 0
\end{array}\right) .
\end{gathered}
$$

It is easy to see by direct calculation that the following recursive relations hold for the powers of the matrix $A_{(\varphi(g), \widehat{\varepsilon}(g)), D S R 4}\left(x^{5}\right)$ :

$$
\begin{aligned}
& \text { 1) }\left(A_{(\varphi(g), \widehat{\varepsilon}(g)), D S R 4}\left(x^{5}\right)\right)^{0}=1_{4-d .} \\
& \text { 2) }\left(A_{(\varphi(g), \widehat{\varepsilon}(g)), D S R 4}\left(x^{5}\right)\right)^{2 n}= \\
& =(-1)^{n-1}\left|\tilde{\theta}\left(g, x^{5}\right)\right|^{2(n-1)}\left(A_{(\varphi(g), \widehat{\varepsilon}(g)), D S R 4}\left(x^{5}\right)\right)^{2}= \\
& =-\left|\tilde{\theta}\left(g, x^{5}\right)\right|^{-2}(-1)^{n}\left|\tilde{\theta}\left(g, x^{5}\right)\right|^{2 n}\left(A_{(\varphi(g), \widehat{\varepsilon}(g)), D S R 4}\left(x^{5}\right)\right)^{2}, n \in N ; \\
& 3)\left(A_{(\varphi(g), \widehat{\varepsilon}(g)), D S R 4}\left(x^{5}\right)\right)^{2 n+1}= \\
& =(-1)^{n}\left|\tilde{\theta}\left(g, x^{5}\right)\right|^{2 n} A_{(\varphi(g), \widehat{\varepsilon}(g)), D S R 4}\left(x^{5}\right), n \in N \cup\{0\}
\end{aligned}
$$

By comparing relations (92) with the corresponding "undeformed" ones valid in SR, it can be immediately seen that the (local) generalizing "anisotropizing deforming" transition SR $\rightarrow$ DSR4 implies the loss of symmetry of all the 
powers of $A_{(\varphi(g), \widehat{\varepsilon}(g)), D S R 4}\left(x^{5}\right)$ (this is due to the loss of symmetry of the 4-d. representation of the infinitesimal generators of $S O(3,1)_{D E F}$ : see Ref. [1]). 
Then, it is possible to evaluate the exponential of $A_{(\varphi(g), \widehat{\varepsilon}(g)), D S R 4}\left(x^{5}\right)$ :

$$
\begin{aligned}
& \exp \left(A_{(\varphi(g), \widehat{\varepsilon}(g)), D S R 4}\left(x^{5}\right)\right)=\sum_{n=0}^{\infty} \frac{1}{n !}\left(A_{(\varphi(g), \widehat{\varepsilon}(g)), D S R 4}\left(x^{5}\right)\right)^{n}= \\
& =1_{4-d .}+\sum_{n=1}^{\infty} \frac{1}{(2 n) !}\left(A_{(\varphi(g), \widehat{\varepsilon}(g)), D S R 4}\left(x^{5}\right)\right)^{2 n}+ \\
& +\sum_{n=0}^{\infty} \frac{1}{(2 n+1) !}\left(A_{(\varphi(g), \widehat{\varepsilon}(g)), D S R 4}\left(x^{5}\right)\right)^{2 n+1}= \\
& =1_{4-d .}-\left|\tilde{\theta}\left(g, x^{5}\right)\right|^{-2}\left(A_{(\varphi(g), \widehat{\varepsilon}(g)), D S R 4}\left(x^{5}\right)\right)^{2} \sum_{n=1}^{\infty}(-1)^{n} \frac{\left|\tilde{\theta}\left(g, x^{5}\right)\right|^{2 n}}{(2 n) !}+ \\
& +A_{(\varphi(g), \widehat{\varepsilon}(g)), D S R 4}\left(x^{5}\right) \sum_{n=0}^{\infty}(-1)^{n} \frac{\left|\tilde{\theta}\left(g, x^{5}\right)\right|^{2 n}}{(2 n+1) !}= \\
& =1_{4-d .}-\left|\tilde{\theta}\left(g, x^{5}\right)\right|^{-2}\left(A_{(\varphi(g), \widehat{\varepsilon}(g)), D S R 4}\left(x^{5}\right)\right)^{2} \sum_{n=1}^{\infty}(-1)^{n} \frac{\left|\tilde{\theta}\left(g, x^{5}\right)\right|^{2 n}}{(2 n) !}+ \\
& +\left|\tilde{\theta}\left(g, x^{5}\right)\right|^{-1} A_{(\varphi(g), \widehat{\varepsilon}(g)), D S R 4}\left(x^{5}\right) \sum_{n=0}^{\infty}(-1)^{n} \frac{\left|\tilde{\theta}\left(g, x^{5}\right)\right|^{2 n+1}}{(2 n+1) !}= \\
& =1_{4-d .}-\left|\tilde{\theta}\left(g, x^{5}\right)\right|^{-2}\left(\cos \left|\tilde{\theta}\left(g, x^{5}\right)\right|-1\right)\left(A_{(\varphi(g), \widehat{\varepsilon}(g)), D S R 4}\left(x^{5}\right)\right)^{2}+ \\
& +\left|\tilde{\theta}\left(g, x^{5}\right)\right|^{-1} \sin \left|\tilde{\theta}\left(g, x^{5}\right)\right| A_{(\varphi(g), \widehat{\varepsilon}(g)), D S R 4}\left(x^{5}\right) .
\end{aligned}
$$


In conclusion, we have, for the finite, deformed (clockwise) true rotation by an angle $\varphi(g)$ about a generic axis $\widehat{\varepsilon}(g)$ of $\widetilde{E}_{3}\left(x^{5}\right)\left(\subset \widetilde{M_{4}}\left(x^{5}\right)\right)$ :

$$
\begin{gathered}
\left(\begin{array}{c}
\left(x^{\prime}\right)_{(g), D S R 4}^{0}\left(\{x\}_{m .}, x^{5}\right) \\
\left(x^{\prime}\right)_{(g), D S R 4}^{1}\left(\{x\}_{m .}, x^{5}\right) \\
\left(x^{\prime}\right)_{(g), D S R 4}^{2}\left(\{x\}_{m .}, x^{5}\right) \\
\left(x^{\prime}\right)_{(g), D S R 4}^{3}\left(\{x\}_{m .}, x^{5}\right)
\end{array}\right)=\left(\begin{array}{c}
\left(x^{0}\right)_{(g), D S R 4}^{\prime}\left(\{x\}_{m .}, x^{5}\right) \\
\left(x^{1}\right)_{(g), D S R 4}^{\prime}\left(\{x\}_{m .}, x^{5}\right) \\
\left(x^{2}\right)_{(g), D S R 4}^{\prime}\left(\{x\}_{m .}, x^{5}\right) \\
\left(x^{3}\right)_{(g), D S R 4}^{\prime}\left(\{x\}_{m .}, x^{5}\right)
\end{array}\right)= \\
=\left(\exp \left(A_{(\varphi(g), \widehat{\varepsilon}(g)), D S R 4}\left(x^{5}\right)\right)\right)\left(\begin{array}{c}
x^{0} \\
x^{1} \\
x^{2} \\
x^{3}
\end{array}\right),
\end{gathered}
$$

with explicit components (where • indicates the usual algebraic multiplication)

$$
\begin{gathered}
\left(x^{\prime}\right)_{(g), D S R 4}^{0}\left(\{x\}_{m .}, x^{5}\right)=\left(x^{0}\right)_{(g), D S R 4}^{\prime}\left(\{x\}_{m .}, x^{5}\right)= \\
\quad=\sum_{\mu=0}^{3}\left(\exp \left(A_{(\varphi(g), \widehat{\varepsilon}(g)), D S R 4}\left(x^{5}\right)\right)\right)_{\mu}^{0} x^{\mu}=x^{0}
\end{gathered}
$$




$$
\begin{aligned}
& \left(x^{\prime}\right)_{(g), D S R 4}^{1}\left(\{x\}_{m .}, x^{5}\right)=\left(x^{1}\right)_{(g), D S R 4}^{\prime}\left(\{x\}_{m .}, x^{5}\right)=\sum_{\mu=0}^{3}\left(\exp \left(A_{(\varphi(g), \widehat{\varepsilon}(g)), D S R 4}\left(x^{5}\right)\right)\right)_{\mu}^{1} x^{\mu}= \\
& =\left[1+\frac{\left(\cos \left|\tilde{\theta}\left(g, x^{5}\right)\right|-1\right)\left(\left|\tilde{\theta}\left(g, x^{5}\right)\right|^{2}-\left(\theta^{1}(g)\right)^{2} b_{2}^{-2}\left(x^{5}\right) b_{3}^{-2}\left(x^{5}\right)\right)}{\left|\tilde{\theta}\left(g, x^{5}\right)\right|^{2}}\right] x^{1}+ \\
& +\frac{\theta^{3}(g) b_{1}^{-2}\left(x^{5}\right)\left|\tilde{\theta}\left(g, x^{5}\right)\right| \sin \left|\tilde{\theta}\left(g, x^{5}\right)\right|}{\left|\tilde{\theta}\left(g, x^{5}\right)\right|^{2}} x^{2}+ \\
& -\frac{\left(\cos \left|\tilde{\theta}\left(g, x^{5}\right)\right|-1\right) \theta^{1}(g) \theta^{2}(g) b_{1}^{-2}\left(x^{5}\right) b_{3}^{-2}\left(x^{5}\right)}{\left|\tilde{\theta}\left(g, x^{5}\right)\right|^{2}} x^{2}+ \\
& -\frac{\theta^{2}(g) b_{1}^{-2}\left(x^{5}\right)\left|\tilde{\theta}\left(g, x^{5}\right)\right| \sin \left|\tilde{\theta}\left(g, x^{5}\right)\right|}{\left|\tilde{\theta}\left(g, x^{5}\right)\right|^{2}} x^{3}+ \\
& +\frac{\left(\cos \left|\tilde{\theta}\left(g, x^{5}\right)\right|-1\right) \theta^{1}(g) \theta^{3}(g) b_{1}^{-2}\left(x^{5}\right) b_{2}^{-2}\left(x^{5}\right)}{\left|\tilde{\theta}\left(g, x^{5}\right)\right|^{2}} x^{3}= \\
& =\frac{1}{\left|\tilde{\theta}\left(g, x^{5}\right)\right|^{2}}\left\{\left[\left|\tilde{\theta}\left(g, x^{5}\right)\right|^{2}+\right.\right. \\
& \left.+\left(\cos \left|\tilde{\theta}\left(g, x^{5}\right)\right|-1\right)\left(\left|\tilde{\theta}\left(g, x^{5}\right)\right|^{2}-\left(\theta^{1}(g)\right)^{2} b_{2}^{-2}\left(x^{5}\right) b_{3}^{-2}\left(x^{5}\right)\right)\right] x^{1}+ \\
& +\left[\theta^{3}(g) b_{1}^{-2}\left(x^{5}\right)\left|\tilde{\theta}\left(g, x^{5}\right)\right| \sin \left|\tilde{\theta}\left(g, x^{5}\right)\right|+\right. \\
& \left.-\left(\cos \left|\tilde{\theta}\left(g, x^{5}\right)\right|-1\right) \theta^{1}(g) \theta^{2}(g) b_{1}^{-2}\left(x^{5}\right) b_{3}^{-2}\left(x^{5}\right)\right] x^{2}+ \\
& -\left[\theta^{2}(g) b_{1}^{-2}\left(x^{5}\right)\left|\tilde{\theta}\left(g, x^{5}\right)\right| \sin \left|\tilde{\theta}\left(g, x^{5}\right)\right|+\right. \\
& \left.\left.+\left(\cos \left|\tilde{\theta}\left(g, x^{5}\right)\right|-1\right) \theta^{1}(g) \theta^{3}(g) b_{1}^{-2}\left(x^{5}\right) b_{2}^{-2}\left(x^{5}\right)\right] x^{3}\right\}
\end{aligned}
$$




$$
\begin{aligned}
& \left(x^{\prime}\right)_{(g), D S R 4}^{2}\left(\{x\}_{m .}, x^{5}\right)=\left(x^{2}\right)_{(g), D S R 4}^{\prime}\left(\{x\}_{m .}, x^{5}\right)=\sum_{\mu=0}^{3}\left(\exp \left(A_{(\varphi(g), \widehat{\varepsilon}(g)), D S R 4}\left(x^{5}\right)\right)\right)_{\mu}^{2} x^{\mu}= \\
& =-\frac{\theta^{3}(g) b_{2}^{-2}\left(x^{5}\right)\left|\tilde{\theta}\left(g, x^{5}\right)\right| \sin \left|\tilde{\theta}\left(g, x^{5}\right)\right|}{\left|\tilde{\theta}\left(g, x^{5}\right)\right|^{2}} x^{1}+ \\
& +\frac{\left(\cos \left|\tilde{\theta}\left(g, x^{5}\right)\right|-1\right) \theta^{1}(g) \theta^{2}(g) b_{2}^{-2}\left(x^{5}\right) b_{3}^{-2}\left(x^{5}\right)}{\left|\tilde{\theta}\left(g, x^{5}\right)\right|^{2}} x^{1}+ \\
& +\left[1+\frac{\left(\cos \left|\tilde{\theta}\left(g, x^{5}\right)\right|-1\right)\left(\left|\tilde{\theta}\left(g, x^{5}\right)\right|^{2}-\left(\theta^{2}(g)\right)^{2} b_{1}^{-2}\left(x^{5}\right) b_{3}^{-2}\left(x^{5}\right)\right)}{\left|\tilde{\theta}\left(g, x^{5}\right)\right|^{2}}\right] x^{2}+ \\
& +\frac{\theta^{1}(g) b_{2}^{-2}\left(x^{5}\right)\left|\tilde{\theta}\left(g, x^{5}\right)\right| \sin \left|\tilde{\theta}\left(g, x^{5}\right)\right|}{\left|\tilde{\theta}\left(g, x^{5}\right)\right|^{2}} x^{3}+ \\
& -\frac{\left(\cos \left|\tilde{\theta}\left(g, x^{5}\right)\right|-1\right) \theta^{2}(g) \theta^{3}(g) b_{1}^{-2}\left(x^{5}\right) b_{2}^{-2}\left(x^{5}\right)}{\left|\tilde{\theta}\left(g, x^{5}\right)\right|^{2}} x^{3}= \\
& =\frac{1}{\left|\tilde{\theta}\left(g, x^{5}\right)\right|^{2}}\left\{-\left[\theta^{3}(g) b_{2}^{-2}\left(x^{5}\right)\left|\tilde{\theta}\left(g, x^{5}\right)\right| \sin \left|\tilde{\theta}\left(g, x^{5}\right)\right|+\right.\right. \\
& \left.+\left(\cos \left|\tilde{\theta}\left(g, x^{5}\right)\right|-1\right) \theta^{1}(g) \theta^{2}(g) b_{2}^{-2}\left(x^{5}\right) b_{3}^{-2}\left(x^{5}\right)\right] x^{1}+ \\
& +\left[\left|\tilde{\theta}\left(g, x^{5}\right)\right|^{2}+\left(\cos \left|\tilde{\theta}\left(g, x^{5}\right)\right|-1\right) \bullet\right. \\
& \text { - } \left.\left(\left|\tilde{\theta}\left(g, x^{5}\right)\right|^{2}-\left(\theta^{2}(g)\right)^{2} b_{1}^{-2}\left(x^{5}\right) b_{3}^{-2}\left(x^{5}\right)\right)\right] x^{2}+ \\
& 46 \\
& +\left[\theta^{1}(g) b_{2}^{-2}\left(x^{5}\right)\left|\tilde{\theta}\left(g, x^{5}\right)\right| \sin \left|\tilde{\theta}\left(g, x^{5}\right)\right|+\right. \\
& \left.\left.-\left(\cos \left|\tilde{\theta}\left(g, x^{5}\right)\right|-1\right) \theta^{2}(g) \theta^{3}(g) b_{1}^{-2}\left(x^{5}\right) b_{2}^{-2}\left(x^{5}\right)\right] x^{3}\right\}
\end{aligned}
$$




$$
\begin{aligned}
& \left(x^{\prime}\right)_{(g), D S R 4}^{3}\left(\{x\}_{m .}, x^{5}\right)=\left(x^{3}\right)_{(g), D S R 4}^{\prime}\left(\{x\}_{m .}, x^{5}\right)=\sum_{\mu=0}^{3}\left(\exp \left(A_{(\varphi(g), \widehat{\varepsilon}(g)), D S R 4}\left(x^{5}\right)\right)\right)_{\mu}^{3} x^{\mu}= \\
& =\frac{\theta^{2}(g) b_{3}^{-2}\left(x^{5}\right)\left|\tilde{\theta}\left(g, x^{5}\right)\right| \sin \left|\tilde{\theta}\left(g, x^{5}\right)\right|}{\left|\tilde{\theta}\left(g, x^{5}\right)\right|^{2}} x^{1}+ \\
& -\frac{\left(\cos \left|\tilde{\theta}\left(g, x^{5}\right)\right|-1\right) \theta^{1}(g) \theta^{3}(g) b_{2}^{-2}\left(x^{5}\right) b_{3}^{-2}\left(x^{5}\right)}{\left|\tilde{\theta}\left(g, x^{5}\right)\right|^{2}} x^{1}+ \\
& -\frac{\theta^{1}(g) b_{3}^{-2}\left(x^{5}\right)\left|\tilde{\theta}\left(g, x^{5}\right)\right| \sin \left|\tilde{\theta}\left(g, x^{5}\right)\right|}{\left|\tilde{\theta}\left(g, x^{5}\right)\right|^{2}} x^{2}+ \\
& +\frac{\left(\cos \left|\tilde{\theta}\left(g, x^{5}\right)\right|-1\right) \theta^{2}(g) \theta^{3}(g) b_{1}^{-2}\left(x^{5}\right) b_{3}^{-2}\left(x^{5}\right)}{\left|\tilde{\theta}\left(g, x^{5}\right)\right|^{2}} x^{2}+ \\
& +\left[1+\frac{\left(\cos \left|\tilde{\theta}\left(g, x^{5}\right)\right|-1\right)\left(\left|\tilde{\theta}\left(g, x^{5}\right)\right|^{2}-\left(\theta^{3}(g)\right)^{2} b_{1}^{-2}\left(x^{5}\right) b_{2}^{-2}\left(x^{5}\right)\right)}{\left|\tilde{\theta}\left(g, x^{5}\right)\right|^{2}}\right] x^{3}= \\
& =\frac{1}{\left|\tilde{\theta}\left(g, x^{5}\right)\right|^{2}}\left\{\left[\theta^{2}(g) b_{3}^{-2}\left(x^{5}\right)\left|\tilde{\theta}\left(g, x^{5}\right)\right| \sin \left|\tilde{\theta}\left(g, x^{5}\right)\right|+\right.\right. \\
& \left.-\left(\cos \left|\tilde{\theta}\left(g, x^{5}\right)\right|-1\right) \theta^{1}(g) \theta^{3}(g) b_{2}^{-2}\left(x^{5}\right) b_{3}^{-2}\left(x^{5}\right)\right] x^{1}+ \\
& -\left[\theta^{1}(g) b_{3}^{-2}\left(x^{5}\right)\left|\tilde{\theta}\left(g, x^{5}\right)\right| \sin \left|\tilde{\theta}\left(g, x^{5}\right)\right|+\right. \\
& \left.+\left(\cos \left|\tilde{\theta}\left(g, x^{5}\right)\right|-1\right) \theta^{2}(g) \theta^{3}(g) b_{1}^{-2}\left(x^{5}\right) b_{3}^{-2}\left(x^{5}\right)\right] x^{2}+ \\
& 47 \\
& +\left[\left|\tilde{\theta}\left(g, x^{5}\right)\right|^{2}+\left(\cos \left|\tilde{\theta}\left(g, x^{5}\right)\right|-1\right) \bullet\right. \\
& \text { - } \left.\left.\left(\left|\tilde{\theta}\left(g, x^{5}\right)\right|^{2}-\left(\theta^{3}(g)\right)^{2} b_{1}^{-2}\left(x^{5}\right) b_{2}^{-2}\left(x^{5}\right)\right)\right] x^{3}\right\} \text {. }
\end{aligned}
$$




\section{FINITE 3-d. DEFORMED BOOSTS IN A GENERIC DIRECTION IN DSR4}

\subsection{Parametric Decomposition}

We want now to derive the finite, deformed pseudorotations (or "boosts") with generic dimensionless parameter ("rapidity") $\rho(g)$ along a generic direction $\widehat{\varepsilon}(g)$ in the physical 3-d. space $\widetilde{E_{3}}\left(x^{5}\right) \subset \widetilde{M_{4}}\left(x^{5}\right)$, by exploiting the form of the infinitesimal generators of the DSR4 chronotopical group $S O(3,1)_{D E F}$. obtained in Ref. [1].

We can proceed in a similar way to the case of finite deformed true rotations treated in the previous Section 5. Thus we have the following (not unique) axial-parametric decomposition:

$$
(\widehat{\varepsilon}(g), \rho(g)) \rightarrow\left(\widehat{x^{1}}, \zeta^{1}(g)\right)\left(\widehat{x^{2}}, \zeta^{2}(g)\right)\left(\widehat{x^{3}}, \zeta^{3}(g)\right)
$$

whence in DSR4 we obtain (- denotes the Euclidean scalar product):

$$
\begin{gathered}
-\rho(g) \widehat{\varepsilon}(g) \cdot \mathbf{K}_{D S R 4}\left(x^{5}\right) \rightarrow \\
\rightarrow-\zeta^{1}(g) K_{D S R 4}^{1}\left(x^{5}\right)-\zeta^{2}(g) K_{D S R 4}^{2}\left(x^{5}\right)-\zeta^{3}(g) K_{D S R 4}^{3}\left(x^{5}\right)= \\
=-\sum_{i=1}^{3} \zeta^{i}(g) K_{D S R 4}^{i}\left(x^{5}\right)
\end{gathered}
$$

(infinitesimal level, any composition order);

$$
\begin{gathered}
\exp \left(-\rho(g) \widehat{\varepsilon}(g) \cdot \mathbf{K}_{D S R 4}\left(x^{5}\right)\right) \rightarrow \\
\rightarrow \exp \left(-\zeta^{1}(g) K_{D S R 4}^{1}\left(x^{5}\right)\right) \times \exp \left(-\zeta^{2}(g) K_{D S R 4}^{2}\left(x^{5}\right)\right) \times \exp \left(-\zeta^{3}(g) K_{D S R 4}^{3}\left(x^{5}\right)\right)
\end{gathered}
$$

(finite level, fixed composition order).

Denoting by $B_{(\rho(g), \widehat{\varepsilon}(g)), D S R 4}\left(x^{5}\right)$ the $4 \times 4$ matrix corresponding to an infinitesimal boost with (infinitesimal) rapidity $\rho(g)$ about the axis $\widehat{\varepsilon}(g)$ 
(matrix belonging a 4-d. representation of the deformed Lorentz algebra $s u(2)_{D E F} \times s u(2)_{D E F}$. , we have to go through the following steps:

$$
\begin{gathered}
B_{(\rho(g), \widehat{\varepsilon}(g)), D S R 4}\left(x^{5}\right) \equiv-\rho(g) \widehat{\varepsilon}(g) \cdot \mathbf{K}_{D S R 4}\left(x^{5}\right) \rightarrow \\
\rightarrow\left(-\zeta^{1}(g) K_{D S R 4}^{1}\left(x^{5}\right)-\zeta^{2}(g) K_{D S R 4}^{2}\left(x^{5}\right)-\zeta^{3}(g) K_{D S R 4}^{3}\left(x^{5}\right)\right) \rightarrow \\
\rightarrow \exp \left(B_{(\rho(g), \widehat{\varepsilon}(g)), D S R 4}\left(x^{5}\right)\right)= \\
=\exp \left(-\zeta^{1}(g) K_{D S R 4}^{1}\left(x^{5}\right)-\zeta^{2}(g) K_{D S R 4}^{2}\left(x^{5}\right)-\zeta^{3}(g) K_{D S R 4}^{3}\left(x^{5}\right)\right)= \\
=\sum_{n=0}^{\infty} \frac{1}{n !}\left(-\zeta^{1}(g) K_{D S R 4}^{1}\left(x^{5}\right)-\zeta^{2}(g) K_{D S R 4}^{2}\left(x^{5}\right)-\zeta^{3}(g) K_{D S R 4}^{3}\left(x^{5}\right)\right)^{n}
\end{gathered}
$$

where $\exp \left(B_{(\rho(g), \widehat{\varepsilon}(g)), D S R 4}\left(x^{5}\right)\right)$ is the $4 \times 4$ matrix corresponding to a finite pseudorotation with finite rapidity $\varphi(g)$ along the axis $\widehat{\varepsilon}(g)$, belonging to a 4-d. representation of $S O(3,1)_{D E F}$, of course.

On account of the explicit form of $\mathbf{K}_{D S R 4}\left(x^{5}\right)$ and of the deformed boost generators (see Subsect. 4.1), we have

$$
\begin{gathered}
B_{(\rho(g), \widehat{\varepsilon}(g)), D S R 4}\left(x^{5}\right)= \\
=\left(\begin{array}{cccc}
0 & -\zeta^{1}(g) b_{0}^{-2}\left(x^{5}\right) & -\zeta^{2}(g) b_{0}^{-2}\left(x^{5}\right) & -\zeta^{3}(g) b_{0}^{-2}\left(x^{5}\right) \\
-\zeta^{1}(g) b_{1}^{-2}\left(x^{5}\right) & 0 & 0 & 0 \\
-\zeta^{2}(g) b_{2}^{-2}\left(x^{5}\right) & 0 & 0 & 0 \\
-\zeta^{3}(g) b_{3}^{-2}\left(x^{5}\right) & 0 & 0 & 0
\end{array}\right),
\end{gathered}
$$


and therefore the corresponding finite form is given by the matrix exponential

$$
\begin{gathered}
\exp \left(B_{(\rho(g), \widehat{\varepsilon}(g)), D S R 4}\left(x^{5}\right)\right)= \\
=\exp \left(\begin{array}{cccc}
0 & -\zeta^{1}(g) b_{0}^{-2}\left(x^{5}\right) & -\zeta^{2}(g) b_{0}^{-2}\left(x^{5}\right) & -\zeta^{3}(g) b_{0}^{-2}\left(x^{5}\right) \\
-\zeta^{1}(g) b_{1}^{-2}\left(x^{5}\right) & 0 & 0 & 0 \\
-\zeta^{2}(g) b_{2}^{-2}\left(x^{5}\right) & 0 & 0 & 0 \\
-\zeta^{3}(g) b_{3}^{-2}\left(x^{5}\right) & 0 & 0 & 0
\end{array}\right) .
\end{gathered}
$$


Therefore, in matrix form, the deformed boost transformation reads

$$
\begin{aligned}
& \left(\begin{array}{c}
\left(x^{\prime}\right)_{(g), D S R 4}^{0}\left(\{x\}_{m .}, x^{5}\right) \\
\left(x^{\prime}\right)_{(g), D S R 4}^{1}\left(\{x\}_{m .}, x^{5}\right) \\
\left(x^{\prime}\right)_{(g), D S R 4}^{2}\left(\{x\}_{m .}, x^{5}\right) \\
\left(x^{\prime}\right)_{(g), D S R 4}^{3}\left(\{x\}_{m .}, x^{5}\right)
\end{array}\right)=\left(\begin{array}{c}
\left(x^{0}\right)_{(g), D S R 4}^{\prime}\left(\{x\}_{m .}, x^{5}\right) \\
\left(x^{1}\right)_{(g), D S R 4}^{\prime}\left(\{x\}_{m .}, x^{5}\right) \\
\left(x^{2}\right)_{(g), D S R 4}^{\prime}\left(\{x\}_{m .}, x^{5}\right) \\
\left(x^{3}\right)_{(g), D S R 4}^{\prime}\left(\{x\}_{m .}, x^{5}\right)
\end{array}\right)= \\
& =\exp \left(B_{(\rho(g), \widehat{\varepsilon}(g)), D S R 4}\left(x^{5}\right)\right)\left(\begin{array}{c}
x^{0} \\
x^{1} \\
x^{2} \\
x^{3}
\end{array}\right) \Leftrightarrow \\
& \Leftrightarrow\left(\begin{array}{c}
\left(x^{\prime}\right)_{(g), D S R 4}^{0}\left(\{x\}_{m .}, x^{5}\right) \\
\left(x^{\prime}\right)_{(g), D S R 4}^{1}\left(\{x\}_{m .}, x^{5}\right) \\
\left(x^{\prime}\right)_{(g), D S R 4}^{2}\left(\{x\}_{m .}, x^{5}\right) \\
\left(x^{\prime}\right)_{(g), D S R 4}^{3}\left(\{x\}_{m .}, x^{5}\right)
\end{array}\right)=\left(\begin{array}{c}
\left(x^{0}\right)_{(g), D S R 4}^{\prime}\left(\{x\}_{m .}, x^{5}\right) \\
\left(x^{1}\right)_{(g), D S R 4}^{\prime}\left(\{x\}_{m .}, x^{5}\right) \\
\left(x^{2}\right)_{(g), D S R 4}^{\prime}\left(\{x\}_{m .}, x^{5}\right) \\
\left(x^{3}\right)_{(g), D S R 4}^{\prime}\left(\{x\}_{m .}, x^{5}\right)
\end{array}\right)= \\
& =\exp \left(\begin{array}{cccc}
0 & -\zeta^{1}(g) b_{0}^{-2}\left(x^{5}\right) & -\zeta^{2}(g) b_{0}^{-2}\left(x^{5}\right) & -\zeta^{3}(g) b_{0}^{-2}\left(x^{5}\right) \\
-\zeta^{1}(g) b_{1}^{-2}\left(x^{5}\right) & 0 & 0 & 0 \\
-\zeta^{2}(g) b_{2}^{-2}\left(x^{5}\right) & 0 & 0 & 0 \\
-\zeta^{3}(g) b_{3}^{-2}\left(x^{5}\right) & 0 & 0 & 0
\end{array}\right)\left(\begin{array}{c}
x^{0} \\
x^{1} \\
x^{2} \\
x^{3}
\end{array}\right)
\end{aligned}
$$

\subsection{Deformed Boost from Velocity Decomposition}

As noticed in Sect. 5, all the possible procedures applicable in these frameworks are equivalent (although they may yield different formal results). Thus, in order to obtain the general form of a finite, deformed boost with generic dimensionless parameter ("rapidity") $\rho(g)$ along a generic direction $\widehat{\varepsilon}(g)$ in 
$\widetilde{E_{3}}\left(x^{5}\right) \subset \widetilde{M_{4}}\left(x^{5}\right)$, let us exploit another approach instead of explicitly calculating $\exp \left(B_{(\rho(g), \widehat{\varepsilon}(g)), D S R 4}\left(x^{5}\right)\right)$.

Consider a generic finite, deformed boost with generic velocity $\mathbf{v}(g)=$ $v(g) \widehat{v}(g) \equiv v(g) \widehat{\varepsilon}(g)$ along a generic direction $\widehat{\varepsilon}(g)$ in $\widetilde{E}_{3}\left(x^{5}\right) \subset \widetilde{M}_{4}\left(x^{5}\right)$. Let us decompose the 3 -vector $\mathbf{x}$ in two components $\mathbf{x}_{\|}(g)$ and $\mathbf{x}_{\perp}(g)$, respectively parallel and orthogonal to $\mathbf{v}(g)$. Here, "parallelism" and "orthogonality" are to be meant in the deformed 3-d. space $\widetilde{E}_{3}\left(x^{5}\right) \subset \widetilde{M_{4}}\left(x^{5}\right)$ (namely according to the deformed scalar product $*$ associated to the 3 -d. metric tensor $\left.-g_{i j, D S R 4}\left(x^{5}\right) \stackrel{\mathrm{ESC}}{=}{ }^{\circ f f} b_{i}^{2}\left(x^{5}\right) \delta_{i j}\right)$. We have

$$
\begin{gathered}
\mathbf{x}_{\|}(g) \equiv \widehat{v}(g)(\widehat{v}(g) * \mathbf{x})=\frac{\mathbf{v}(g)}{|\mathbf{v}(g)|_{*}^{2}}(\mathbf{v}(g) * \mathbf{x})=\frac{\mathbf{v}(g)}{\mathbf{v}(g) * \mathbf{v}(g)}(\mathbf{v}(g) * \mathbf{x})= \\
=\frac{\sum_{i=1}^{3} b_{i}^{2}\left(x^{5}\right) v^{i}(g) x^{i}}{\sum_{i=1}^{3} b_{i}^{2}\left(x^{5}\right)\left(v^{i}(g)\right)^{2}} \mathbf{v}(g) \stackrel{\text { in DSR4: } \tilde{\beta}(g) \equiv\left(\frac{\mathbf{v}(g)}{\mathbf{u}}\right) \neq \frac{\mathbf{v}(g)}{u}}{\neq} \widehat{\widetilde{\beta}}(g)(\widetilde{\widetilde{\beta}}(g) * \mathbf{x})= \\
=\frac{\tilde{\beta}(g)}{|\tilde{\beta}(g)|_{*}^{2}}(\tilde{\beta}(g) * \mathbf{x})=\frac{\tilde{\beta}(g)}{\tilde{\beta}(g) * \tilde{\beta}(g)}(\tilde{\beta}(g) * \mathbf{x})= \\
=\frac{\sum_{i=1}^{3} b_{i}^{2}\left(x^{5}\right) \widetilde{\beta}^{i}(g) x^{i}}{\sum_{i=1}^{3} b_{i}^{2}\left(x^{5}\right)\left(\widetilde{\beta}^{i}(g)\right)^{2}} \tilde{\beta}(g) ;
\end{gathered}
$$




$$
x_{\|}^{i}(g) \equiv \frac{\sum_{k=1}^{3} b_{k}^{2}\left(x^{5}\right) v^{k}(g) x^{k}}{\sum_{k=1}^{3} b_{k}^{2}\left(x^{5}\right)\left(v^{k}(g)\right)^{2}} v^{i} \neq
$$$$
\text { in DSR } 4: \tilde{\beta}(g) \equiv\left(\frac{\mathbf{v}(g)}{\mathbf{u}}\right) \neq \frac{\mathbf{v}(g)}{u} \frac{\sum_{k=1}^{3} b_{k}^{2}\left(x^{5}\right) \widetilde{\beta}^{k}(g) x^{k}}{\sum_{k=1}^{3} b_{k}^{2}\left(x^{5}\right)\left(\widetilde{\beta}^{k}(g)\right)^{2}} \widetilde{\beta}^{i}(g), \forall i=1,2,3
$$

$$
\begin{gathered}
\mathbf{x}_{\perp}(g) \equiv \mathbf{x}-\mathbf{x}_{\|}(g)=\mathbf{x}-\frac{\sum_{i=1}^{3} b_{i}^{2}\left(x^{5}\right) v^{i}(g) x^{i}}{\sum_{i=1}^{3} b_{i}^{2}\left(x^{5}\right)\left(v^{i}(g)\right)^{2}} \mathbf{v}(g) \neq \\
\begin{array}{r}
\text { in DSR } 4: \tilde{\beta}(g) \equiv\left(\frac{\mathbf{v}(g)}{\mathbf{u}}\right) \neq \frac{\mathbf{v}(g)}{u} \\
\neq
\end{array}-\frac{\sum_{i=1}^{3} b_{i}^{2}\left(x^{5}\right) \widetilde{\beta}^{i}(g) x^{i}}{\sum_{i=1}^{3} b_{i}^{2}\left(x^{5}\right)\left(\widetilde{\beta}^{i}(g)\right)^{2}} \tilde{\beta}(g) ; \\
x_{\perp}^{i}(g) \equiv x^{i}-\frac{\sum_{k=1}^{3} b_{k}^{2}\left(x^{5}\right) v^{k}(g) x^{k}}{\sum_{k=1}^{3} b_{k}^{2}\left(x^{5}\right)\left(v^{k}(g)\right)^{2}} v^{i}(g) \neq
\end{gathered}
$$

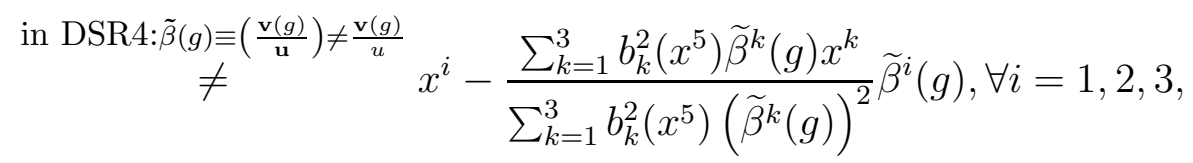


where

$$
\begin{gathered}
\tilde{\beta}(g) \equiv \frac{\mathbf{v}(g)}{\mathbf{u}}= \\
=\left(\frac{v^{1}(g) b_{1}\left(x^{5}\right)}{c b_{0}\left(x^{5}\right)} \widehat{x}, \frac{v^{2}(g) b_{2}\left(x^{5}\right)}{c b_{0}\left(x^{5}\right)} \widehat{y}, \frac{v^{3}(g) b_{3}\left(x^{5}\right)}{c b_{0}\left(x^{5}\right)} \widehat{z}\right) .
\end{gathered}
$$

On account of the form of a finite, deformed boost along a coordinate axis (see Eq. (47)), a finite, deformed boost with generic (finite) velocity $\mathbf{v}(g)$ in a generic direction $\widehat{v}(g)$ is therefore given by (see Ref. [10]) (·denotes, as before, Euclidean 3-d. scalar product)

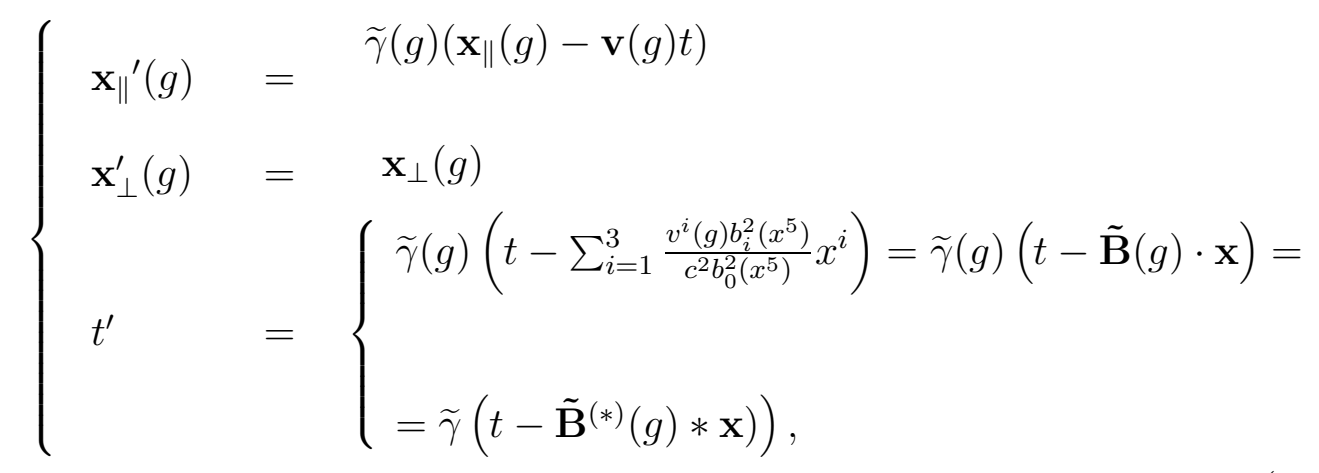

where

$$
\begin{gathered}
\widetilde{\gamma}(g) \equiv(1-\tilde{\beta}(g) \cdot \tilde{\beta}(g))^{-1 / 2}=\left(1-\tilde{\beta}^{(*)}(g) * \tilde{\beta}^{(*)}(g)\right)^{-1 / 2}= \\
=\left(1-\left(\frac{v^{1}(g) b_{1}\left(x^{5}\right)}{c b_{0}\left(x^{5}\right)}\right)^{2}-\left(\frac{v^{2}(g) b_{2}\left(x^{5}\right)}{c b_{0}\left(x^{5}\right)}\right)^{2}-\left(\frac{v^{3}(g) b_{3}\left(x^{5}\right)}{c b_{0}\left(x^{5}\right)}\right)^{2}\right)^{-1 / 2}
\end{gathered}
$$




$$
\begin{gathered}
\tilde{\beta}^{(*)}(g) \equiv \frac{\mathbf{v}(g)}{\mathbf{w}}= \\
=\left(\frac{v^{1}(g)}{c b_{0}\left(x^{5}\right)} \widehat{x}, \frac{v^{2}(g)}{c b_{0}\left(x^{5}\right)} \widehat{y}, \frac{v^{3}(g)}{c b_{0}\left(x^{5}\right)} \widehat{z}\right)=\frac{1}{c b_{0}\left(x^{5}\right)} \mathbf{v}(g) ; \\
\tilde{\mathbf{B}}(g) \equiv \frac{\mathbf{v}(g)}{\mathbf{u}^{2}}= \\
=\left(\frac{v^{1}(g) b_{1}^{2}\left(x^{5}\right)}{c^{2} b_{0}^{2}\left(x^{5}\right)} \widehat{x}, \frac{v^{2}(g) b_{2}^{2}\left(x^{5}\right)}{c^{2} b_{0}^{2}\left(x^{5}\right)} \widehat{y}, \frac{v^{3}(g) b_{3}^{2}\left(x^{5}\right)}{c^{2} b_{0}^{2}\left(x^{5}\right)} \widehat{z}\right) ; \\
\tilde{\mathbf{B}}^{(*)}(g) \equiv \frac{\mathbf{v}(g)}{\mathbf{w}^{2}}=\frac{1}{c^{2} b_{0}^{2}\left(x^{5}\right)} \mathbf{v}(g),
\end{gathered}
$$

and $\mathbf{w}$ is the (unphysical) isotropic maximal causal velocity (see Ref. [10])

$$
\mathbf{w} \equiv c b_{0}\left(x^{5}\right)(\widehat{x}, \widehat{y}, \widehat{z}) .
$$

From the definitions (110) and (113) of $\tilde{\beta}(g)$ and $\tilde{\beta}^{(*)}(g)$ it follows easily ${ }^{13}$

$$
|\mathbf{v}(g)|_{*}^{2} \equiv \sum_{k=1}^{3} b_{i}^{2}\left(x^{5}\right)\left(v^{i}(g)\right)^{2}=c^{2} b_{0}^{2}\left(x^{5}\right)|\tilde{\beta}(g)|^{2} ;
$$

\footnotetext{
${ }^{13}$ Indeed in DSR4, as it may be easily verified, one has

$$
\left|\tilde{\beta}^{(*)}(g)\right|_{*}^{2}=|\tilde{\beta}(g)|^{2}
$$

and

$$
\begin{gathered}
\left|\mathbf{w}\left(x^{5}\right)\right|_{*}^{2}=\sum_{k=1}^{3} b_{i}^{2}\left(x^{5}\right)\left(w^{i}\left(x^{5}\right)\right)^{2}=c^{2} b_{0}^{2}\left(x^{5}\right) \sum_{k=1}^{3} b_{i}^{2}\left(x^{5}\right) \\
\left|\mathbf{u}\left(x^{5}\right)\right|^{2}=\sum_{k=1}^{3}\left(u^{i}\left(x^{5}\right)\right)^{2}=c^{2} b_{0}^{2}\left(x^{5}\right) \sum_{k=1}^{3} b_{i}^{-2}\left(x^{5}\right),
\end{gathered}
$$
}




$$
|\mathbf{v}(g)|_{*}^{2} \equiv \sum_{k=1}^{3} b_{i}^{2}\left(x^{5}\right)\left(v^{i}(g)\right)^{2}=c^{2} b_{0}^{2}\left(x^{5}\right)\left|\tilde{\beta}^{(*)}(g)\right|_{*}^{2} \text {. }
$$

In terms of the trivector $\mathbf{x}=\mathbf{x}_{\|}(g)+\mathbf{x}_{\perp}(g)$, the deformed boost (111) in a generic direction $\widehat{v}(g)$ reads therefore

$$
\begin{aligned}
& \mathbf{x}^{\prime}(g)=\mathbf{x}_{\|}^{\prime}(g)+\mathbf{x}_{\perp}^{\prime}(g)=\left\{\begin{array}{l}
\mathbf{x}+(\widetilde{\gamma}(g)-1) \frac{\mathbf{v}(g)}{|\mathbf{v}(g)|_{*}^{2}}(\mathbf{v}(g) * \mathbf{x})-\widetilde{\gamma}(g) \mathbf{v}(g) t= \\
=\mathbf{x}+(\widetilde{\gamma}(g)-1) \frac{\sum_{k=1}^{3} b_{k}^{2}\left(x^{5}\right) v^{k}(g) x^{k}}{\sum_{k=1}^{3} b_{k}^{2}\left(x^{5}\right)\left(v^{k}(g)\right)^{2}} \mathbf{v}(g)+ \\
-\widetilde{\gamma}(g) \mathbf{v}(g) t ;
\end{array}\right. \\
& t^{\prime}(g)=\left\{\begin{array}{l}
\widetilde{\gamma}(g)(t-\tilde{\mathbf{B}}(g) \cdot \mathbf{x})=\widetilde{\gamma}(g)\left(t-\tilde{\mathbf{B}}^{(*)}(g) * \mathbf{x}\right)= \\
=\widetilde{\gamma}(g)\left(t-\sum_{k=1}^{3} \frac{v^{k}(g) b_{k}^{2}\left(x^{5}\right)}{c^{2} b_{0}^{2}\left(x^{5}\right)} x^{k}\right)
\end{array}\right.
\end{aligned}
$$

or (as usual $x^{0} \equiv c t$, and $\bullet$ now denotes usual algebraic multiplication) so that, in general

$$
\left|\mathbf{w}\left(x^{5}\right)\right|_{*}^{2} \neq\left|\mathbf{u}\left(x^{5}\right)\right|^{2}
$$


$(\forall i=1,2,3)$ :

$$
\begin{gathered}
\left(x^{\prime}\right)_{(g), D S R 4}^{0}\left(\{x\}_{m .}, x^{5}\right)=\left(x^{0}\right)_{(g), D S R 4}^{\prime}\left(\{x\}_{m .}, x^{5}\right)= \\
=\widetilde{\gamma}(g)\left(x^{0}-\sum_{k=1}^{3} \frac{v^{k}(g) b_{k}^{2}\left(x^{5}\right)}{c b_{0}^{2}\left(x^{5}\right)} x^{k}\right)= \\
=\left(1-\left(\frac{v^{1}(g) b_{1}\left(x^{5}\right)}{c b_{0}\left(x^{5}\right)}\right)^{2}-\left(\frac{v^{2}(g) b_{2}\left(x^{5}\right)}{c b_{0}\left(x^{5}\right)}\right)^{2}-\left(\frac{v^{3}(g) b_{3}\left(x^{5}\right)}{c b_{0}\left(x^{5}\right)}\right)^{2}\right)^{-1 / 2} \\
\bullet\left(x^{0}-\sum_{k=1}^{3} \frac{v^{k}(g) b_{k}^{2}\left(x^{5}\right)}{c b_{0}^{2}\left(x^{5}\right)} x^{k}\right)
\end{gathered}
$$




$$
\begin{gathered}
\left(x^{\prime}\right)_{(g), D S R 4}^{i}\left(\{x\}_{m .}, x^{5}\right)=\left(x^{i}\right)_{(g), D S R 4}^{\prime}\left(\{x\}_{m .}, x^{5}\right)= \\
=x^{i}+(\widetilde{\gamma}(g)-1) \frac{\sum_{k=1}^{3} b_{k}^{2}\left(x^{5}\right) v^{k}(g) x^{k}}{\sum_{k=1}^{3} b_{k}^{2}\left(x^{5}\right)\left(v^{k}(g)\right)^{2}} v^{i}(g)-\widetilde{\gamma}(g) \frac{v^{i}(g)}{c} x^{0}= \\
=x^{i}+ \\
+\left(\left(1-\left(\frac{v^{1}(g) b_{1}\left(x^{5}\right)}{c b_{0}\left(x^{5}\right)}\right)^{2}-\left(\frac{v^{2}(g) b_{2}\left(x^{5}\right)}{c b_{0}\left(x^{5}\right)}\right)^{2}-\left(\frac{v^{3}(g) b_{3}\left(x^{5}\right)}{c b_{0}\left(x^{5}\right)}\right)^{2}\right)^{-1 / 2}-1\right) \\
\bullet \frac{\sum_{k=1}^{3} b_{k}^{2}\left(x^{5}\right) v^{k}(g) x^{k}}{\sum_{k=1}^{3} b_{k}^{2}\left(x^{5}\right)\left(v^{k}(g)\right)^{2}} v^{i}(g)+ \\
-\frac{1}{c}\left(1-\left(\frac{v^{1}(g) b_{1}\left(x^{5}\right)}{c b_{0}\left(x^{5}\right)}\right)^{2}-\left(\frac{v^{2}(g) b_{2}\left(x^{5}\right)}{c b_{0}\left(x^{5}\right)}\right)^{2}-\left(\frac{v^{3}(g) b_{3}\left(x^{5}\right)}{c b_{0}\left(x^{5}\right)}\right)^{2}\right)^{-1 / 2} x^{0} v^{i}(g) .
\end{gathered}
$$

Different explicit forms of a finite, deformed boost with generic velocity $\mathbf{v}(g)=v(g) \widehat{v}(g) \equiv v(g) \widehat{\varepsilon}(g)$ along a generic direction $\widehat{\varepsilon}(g)$ in $\widetilde{E}_{3}\left(x^{5}\right) \subset$ $\widetilde{M_{4}}\left(x^{5}\right)$, and consequently of $\exp \left(B_{(\rho(g), \widehat{\varepsilon}(g)), D S R 4}\left(x^{5}\right)\right)$, can be obtained by exploiting Eqs. (113)-(115).

Notice that the lack of symmetry properties of the $4 \times 4$ matrices representing deformed boosts is obviously related to the "anisotropizing deforming" character of the DSR4 generalization of SR.

\subsection{Parametric Change of Basis for a Deformed Boost in a Generic Direction}

On account of Eqs. (55)-(57), which relate, through the use of definition (44), the dimensionless parameter basis of deformed rapidities $\left\{\zeta^{i}(g)\right\},\left\{\tilde{\zeta}^{i}(g)\right\}$ 
and the dimensional parameter basis of deformed boost velocities $\left\{v^{i}(g)\right\}$, one gets (ESC off)

$$
\left\{\begin{array}{l}
I) \quad \frac{v^{i}(g) b_{i}\left(x^{5}\right)}{c b_{0}\left(x^{5}\right)} \equiv \widetilde{\beta}^{i}(g)=b_{i}\left(x^{5}\right) \widetilde{\beta}^{i(*)}(g)= \\
=\operatorname{tgh}\left(\zeta_{i}(g) b_{0}^{-1}\left(x^{5}\right) b_{i}^{-1}\left(x^{5}\right)\right)=\operatorname{tgh}\left(\widetilde{\zeta}_{i}(g)\right) ; \\
I I) \quad\left(1-\frac{b_{i}^{2}\left(x^{5}\right)}{c^{2} b_{0}^{2}\left(x^{5}\right)}\left(v^{i}(g)\right)^{2}\right)^{-1 / 2}=\left(1-\left(\widetilde{\beta}^{i}(g)\right)^{2}\right)^{-1 / 2}= \\
=\left(1-b_{i}^{2}\left(x^{5}\right)\left(\widetilde{\beta}^{i(*)}(g)\right)^{2}\right)^{-1 / 2} \equiv \widetilde{\gamma}^{i}(g)= \\
=\cosh \left(\zeta_{i}(g) b_{0}^{-1}\left(x^{5}\right) b_{i}^{-1}\left(x^{5}\right)\right)=\cosh \left(\widetilde{\zeta}_{i}(g)\right),
\end{array}\right.
$$

and therefore

$$
\begin{gathered}
\tilde{\gamma}(g) \equiv \\
\equiv\left(1-\frac{1}{c^{2} b_{0}^{2}\left(x^{5}\right)} \sum_{k=1}^{3}\left(v^{i}(g)\right)^{2} b_{i}^{2}\left(x^{5}\right)\right)^{-1 / 2}=\left(1-\sum_{i=1}^{3}\left(\widetilde{\beta}^{i}(g)\right)^{2}\right)^{-1 / 2}= \\
=\left(1-\sum_{i=1}^{3} b_{i}^{2}\left(x^{5}\right)\left(\widetilde{\beta}^{i(*)}(g)\right)^{2}\right)^{-1 / 2}=\left(1-\sum_{i=1}^{3}\left(\operatorname{tgh}\left(\zeta_{i}(g) b_{0}^{-1}\left(x^{5}\right) b_{i}^{-1}\left(x^{5}\right)\right)\right)^{2}\right)^{-1 / 2}= \\
=\left(1-\sum_{i=1}^{3}\left(\operatorname{tgh}\left(\widetilde{\zeta}_{i}(g)\right)\right)^{2}\right)^{-1 / 2} ;
\end{gathered}
$$




$$
\begin{aligned}
& \frac{\left(\widetilde{\beta^{i}}(g)\right)^{2}}{|\tilde{\beta}(g)|^{2}}=\frac{b_{i}^{2}\left(x^{5}\right)\left(\widetilde{\beta}^{(*)}(g)\right)^{2}}{\left|\tilde{\beta}^{(*)}(g)\right|_{*}^{2}}= \\
& =\frac{\left(\widetilde{\beta^{i}}(g)\right)^{2}}{\tilde{\beta}(g) \cdot \tilde{\beta}(g)}=\frac{b_{i}^{2}\left(x^{5}\right)\left(\widetilde{\beta}^{(*)}(g)\right)^{2}}{\tilde{\beta^{(*)}}(g) * \tilde{\beta}^{(*)}(g)}= \\
& =\frac{\left(\widetilde{\beta^{i}}(g)\right)^{2}}{\sum_{k=1}^{3}\left(\widetilde{\beta^{k}}(g)\right)^{2}}=\frac{b_{i}^{2}\left(x^{5}\right)\left({\widetilde{\beta^{i}}}^{(*)}(g)\right)^{2}}{\sum_{k=1}^{3} b_{k}^{2}\left(x^{5}\right)\left({\widetilde{\beta^{k}}}^{(*)}(g)\right)^{2}}= \\
& =\frac{\left(\operatorname{tgh}\left(\zeta_{i}(g) b_{0}^{-1}\left(x^{5}\right) b_{i}^{-1}\left(x^{5}\right)\right)\right)^{2}}{\sum_{k=1}^{3}\left(\operatorname{tgh}\left(\zeta_{k}(g) b_{0}^{-1}\left(x^{5}\right) b_{k}^{-1}\left(x^{5}\right)\right)\right)^{2}}= \\
& =\frac{\left(\operatorname{tgh}\left(\widetilde{\zeta}_{i}(g)\right)\right)^{2}}{\sum_{k=1}^{3}\left(\operatorname{tgh}\left(\widetilde{\zeta}_{k}(g)\right)\right)^{2}}
\end{aligned}
$$




$$
\begin{gathered}
\frac{\widetilde{\beta^{i}}(g) \widetilde{\beta^{j}}(g)}{|\tilde{\beta}(g)|^{2}}=\frac{b_{i}\left(x^{5}\right) b_{j}\left(x^{5}\right) \widetilde{\beta}^{(*)}(g) \widetilde{\beta}^{(*)}(g)}{\left|\tilde{\beta}^{(*)}(g)\right|_{*}^{2}}= \\
=\frac{\widetilde{\beta^{i}}(g) \widetilde{\beta^{j}}(g)}{\tilde{\beta}(g) \cdot \tilde{\beta}(g)}=\frac{b_{i}\left(x^{5}\right) b_{j}\left(x^{5}\right) \widetilde{\beta}^{(*)}(g) \widetilde{\beta^{j}}(\mathrm{*})}{\tilde{\beta}^{(*)}(g) * \tilde{\beta}^{(*)}(g)}= \\
=\frac{\widetilde{\beta^{i}}(g) \widetilde{\beta^{j}}(g)}{\sum_{k=1}^{3}\left(\widetilde{\beta}^{k}(g)\right)^{2}}=\frac{b_{i}\left(x^{5}\right) b_{j}\left(x^{5}\right) \widetilde{\beta}^{(*)}(g) \widetilde{\beta}^{(*)}(g)}{\sum_{k=1}^{3} b_{k}^{2}\left(x^{5}\right)\left(\widetilde{\beta}^{k(*)}(g)\right)^{2}}= \\
=\frac{\left(\operatorname{tgh}\left(\zeta_{i}(g) b_{0}^{-1}\left(x^{5}\right) b_{i}^{-1}\left(x^{5}\right)\right)\right)\left(\operatorname{tgh}\left(\zeta_{j}(g) b_{0}^{-1}\left(x^{5}\right) b_{j}^{-1}\left(x^{5}\right)\right)\right)}{\sum_{k=1}^{3}\left(\operatorname{tgh}\left(\zeta_{k}(g) b_{0}^{-1}\left(x^{5}\right) b_{k}^{-1}\left(x^{5}\right)\right)\right)^{2}}= \\
=\frac{\left(\operatorname{tgh}\left(\widetilde{\zeta}_{i}(g)\right)\right)\left(\operatorname{tgh}\left(\widetilde{\zeta}_{j}(g)\right)\right)}{\sum_{k=1}^{3}\left(\operatorname{tgh}\left(\widetilde{\zeta}_{k}(g)\right)\right)^{2}} ;
\end{gathered}
$$




$$
\begin{gathered}
\tilde{\beta}^{2} \equiv|\tilde{\beta}|^{2}=\left|\tilde{\beta}^{(*)}\right|_{*}^{2}=\tilde{\beta}(g) \cdot \tilde{\beta}(g)= \\
=\tilde{\beta}^{(*)}(g) * \tilde{\beta}^{(*)}(g)= \\
=\sum_{k=1}^{3}\left(\widetilde{\beta}^{k}(g)\right)^{2}=\sum_{k=1}^{3} b_{k}^{2}\left(x^{5}\right)\left(\widetilde{\beta}^{k(*)}(g)\right)^{2}= \\
=\sum_{k=1}^{3}\left(t g h\left(\zeta_{k}(g) b_{0}^{-1}\left(x^{5}\right) b_{k}^{-1}\left(x^{5}\right)\right)\right)^{2}= \\
=\sum_{k=1}^{3}\left(\operatorname{tgh}\left(\widetilde{\zeta}_{k}(g)\right)\right)^{2} .
\end{gathered}
$$

The above relations between the sets $\left\{\zeta^{i}(g)\right\}_{i=1,2,3},\left\{\tilde{\zeta}^{i}(g)\right\}_{i=1,2,3}$ and $\left\{v^{i}(g)\right\}_{i=1,2,3}$ express the change of parametric base for deformed boosts in DSR4, from the dimensional parameter basis of deformed boost velocities to the dimensionless parameter basis of deformed rapidities, By means of Eqs. (122)-(125) one can therefore write $\exp \left(B_{(\rho(g), \widehat{\varepsilon}(g)), D S R 4}\left(x^{5}\right)\right)$ (derived in the previous Subsection) in terms of rapidities. We have for instance, in terms of the effective deformed rapidities $\widetilde{\zeta}^{i}\left(g, x^{5}\right)$ defined by Eq. (44),

1) $\left(\exp \left(B_{(\rho(g), \widehat{v}(g) \equiv \widehat{\varepsilon}(g)), D S R 4}\left(x^{5}\right)\right)\right)_{0}^{0}=\left(1-\sum_{i=1}^{3}\left(\operatorname{tgh}\left(\tilde{\zeta}^{i}\left(g, x^{5}\right)\right)\right)^{2}\right)^{-1 / 2}$; 


$$
\begin{aligned}
& \text { 2) }\left(\exp \left(B_{(\rho(g), \widehat{v}(g) \equiv \widehat{\varepsilon}(g)), D S R 4}\left(x^{5}\right)\right)\right)_{m}^{0}= \\
& =-\left(1-\sum_{i=1}^{3}\left(\operatorname{tgh}\left(\tilde{\zeta}^{i}\left(g, x^{5}\right)\right)\right)^{2}\right)^{-1 / 2} \frac{b_{m}\left(x^{5}\right)}{b_{0}\left(x^{5}\right)} \operatorname{tgh}\left(\tilde{\zeta}^{m}\left(g, x^{5}\right)\right), \forall m=1,2,3 \\
& \text { 3) }\left(\exp \left(B_{(\rho(g), \widehat{v}(g) \equiv \widehat{\varepsilon}(g)), D S R 4}\left(x^{5}\right)\right)\right)_{0}^{m}= \\
& =-\left(1-\sum_{i=1}^{3}\left(\operatorname{tgh}\left(\tilde{\zeta}^{i}\left(g, x^{5}\right)\right)\right)^{2}\right)^{-1 / 2} \frac{b_{0}\left(x^{5}\right)}{b_{m}\left(x^{5}\right)} \operatorname{tgh}\left(\tilde{\zeta}^{m}\left(g, x^{5}\right)\right), \forall m=1,2,3 \\
& \text { 4) }\left(\exp \left(B_{(\rho(g), \widehat{v}(g) \equiv \widehat{\varepsilon}(g)), D S R 4}\left(x^{5}\right)\right)\right)_{m}^{m}= \\
& =1+\left(\left(1-\sum_{i=1}^{3}\left(\operatorname{tgh}\left(\tilde{\zeta}^{i}\left(g, x^{5}\right)\right)\right)^{2}\right)^{-1 / 2}-1\right) \frac{\left(\operatorname{tgh}\left(\tilde{\zeta}^{m}\left(g, x^{5}\right)\right)\right)^{2}}{\sum_{k=1}^{3}\left(\operatorname{tgh}\left(\tilde{\zeta}^{k}\left(g, x^{5}\right)\right)\right)^{2}}, \forall m=1,2,3 \\
& \text { 5) }\left(\exp \left(B_{(\rho(g), \widehat{v}(g) \equiv \widehat{\varepsilon}(g)), D S R 4}\left(x^{5}\right)\right)\right)_{2}^{1}= \\
& =\left(\left(1-\sum_{i=1}^{3}\left(\operatorname{tgh}\left(\tilde{\zeta}^{i}\left(g, x^{5}\right)\right)\right)^{2}\right)^{-1 / 2}-1\right) \frac{b_{2}\left(x^{5}\right)}{b_{1}\left(x^{5}\right)} \frac{\left(\operatorname{tgh}\left(\tilde{\zeta}^{1}\left(g, x^{5}\right)\right)\right)\left(\operatorname{tgh}\left(\tilde{\zeta}^{2}\left(g, x^{5}\right)\right)\right)}{\sum_{k=1}^{3}\left(\operatorname{tgh}\left(\tilde{\zeta}^{k}\left(g, x^{5}\right)\right)\right)^{2}}
\end{aligned}
$$


6) $\left(\exp \left(B_{(\rho(g), \widehat{v}(g) \equiv \widehat{\varepsilon}(g)), D S R 4}\left(x^{5}\right)\right)\right)_{3}^{1}=$

$$
=\left(\left(1-\sum_{i=1}^{3}\left(\operatorname{tgh}\left(\tilde{\zeta}^{i}\left(g, x^{5}\right)\right)\right)^{2}\right)^{-1 / 2}-1\right) \frac{b_{3}\left(x^{5}\right)}{b_{1}\left(x^{5}\right)} \frac{\left(\operatorname{tgh}\left(\tilde{\zeta}^{1}\left(g, x^{5}\right)\right)\right)\left(\operatorname{tgh}\left(\tilde{\zeta}^{3}\left(g, x^{5}\right)\right)\right)}{\sum_{k=1}^{3}\left(\operatorname{tgh}\left(\tilde{\zeta}^{k}\left(g, x^{5}\right)\right)\right)^{2}}
$$

7) $\left(\exp \left(B_{(\rho(g), \widehat{v}(g) \equiv \widehat{\varepsilon}(g)), D S R 4}\left(x^{5}\right)\right)\right)_{1}^{2}=$

$$
=\left(\left(1-\sum_{i=1}^{3}\left(\operatorname{tgh}\left(\tilde{\zeta}^{i}\left(g, x^{5}\right)\right)\right)^{2}\right)^{-1 / 2}-1\right) \frac{b_{1}\left(x^{5}\right)}{b_{2}\left(x^{5}\right)} \frac{\left(\operatorname{tgh}\left(\tilde{\zeta}^{1}\left(g, x^{5}\right)\right)\right)\left(\operatorname{tgh}\left(\tilde{\zeta}^{2}\left(g, x^{5}\right)\right)\right)}{\sum_{k=1}^{3}\left(\operatorname{tgh}\left(\tilde{\zeta}^{k}\left(g, x^{5}\right)\right)\right)^{2}}
$$

8) $\left(\exp \left(B_{(\rho(g), \widehat{v}(g) \equiv \widehat{\varepsilon}(g)), D S R 4}\left(x^{5}\right)\right)\right)_{3}^{2}=$

$$
\begin{gathered}
=\left(\left(1-\sum_{i=1}^{3}\left(\operatorname{tgh}\left(\tilde{\zeta}^{i}\left(g, x^{5}\right)\right)\right)^{2}\right)^{-1 / 2}-1\right) \frac{b_{3}\left(x^{5}\right)}{b_{2}\left(x^{5}\right)} \frac{\left(\operatorname{tgh}\left(\tilde{\zeta}^{2}\left(g, x^{5}\right)\right)\right)\left(\operatorname{tgh}\left(\tilde{\zeta}^{3}\left(g, x^{5}\right)\right)\right)}{\sum_{k=1}^{3}\left(\operatorname{tgh}\left(\tilde{\zeta}^{k}\left(g, x^{5}\right)\right)\right)^{2}} ; \\
9) \quad\left(\exp \left(B_{(\rho(g), \widehat{v}(g) \equiv \widehat{\varepsilon}(g)), \operatorname{DSR} 4}\left(x^{5}\right)\right)\right)_{1}^{3}= \\
=\left(\left(1-\sum_{i=1}^{3}\left(\operatorname{tgh}\left(\tilde{\zeta}^{i}\left(g, x^{5}\right)\right)\right)^{2}\right)^{-1 / 2}-1\right) \frac{b_{1}\left(x^{5}\right)}{b_{3}\left(x^{5}\right)} \frac{\left(\operatorname{tgh}\left(\tilde{\zeta}^{1}\left(g, x^{5}\right)\right)\right)\left(\operatorname{tgh}\left(\tilde{\zeta}^{3}\left(g, x^{5}\right)\right)\right)}{\sum_{k=1}^{3}\left(\operatorname{tgh}\left(\tilde{\zeta}^{k}\left(g, x^{5}\right)\right)\right)^{2}}
\end{gathered}
$$




$$
\begin{gathered}
10) \quad\left(\exp \left(B_{(\rho(g), \widehat{v}(g) \equiv \widehat{\varepsilon}(g)), D S R 4}\left(x^{5}\right)\right)\right)_{2}^{3}= \\
=\left(\left(1-\sum_{i=1}^{3}\left(\operatorname{tgh}\left(\tilde{\zeta}^{i}\left(g, x^{5}\right)\right)\right)^{2}\right)^{-1 / 2}-1\right) \frac{b_{2}\left(x^{5}\right)}{b_{3}\left(x^{5}\right)} \frac{\left(\operatorname{tgh}\left(\tilde{\zeta}^{2}\left(g, x^{5}\right)\right)\right)\left(\operatorname{tgh}\left(\tilde{\zeta}^{3}\left(g, x^{5}\right)\right)\right)}{\sum_{k=1}^{3}\left(\operatorname{tgh}\left(\tilde{\zeta}^{k}\left(g, x^{5}\right)\right)\right)^{2}} ;
\end{gathered}
$$




$$
\begin{gathered}
\left(x^{\prime}\right)_{(g), D S R 4}^{0}\left(\{x\}_{m .}, x^{5}\right)=\left(x^{0}\right)_{(g), D S R 4}^{\prime}\left(\{x\}_{m .}, x^{5}\right)= \\
=\sum_{\mu=0}^{3}\left(\exp \left(B_{(\rho(g), \widehat{v}(g) \equiv \widehat{\varepsilon}(g)), D S R 4}\left(x^{5}\right)\right)\right)_{\mu}^{0} x^{\mu}= \\
=\left[\left(1-\sum_{i=1}^{3}\left(\operatorname{tgh}\left(\tilde{\zeta}_{i}\left(g, x^{5}\right)\right)\right)^{2}\right)^{-1 / 2}\right] x^{0}+ \\
-\left[\left(1-\sum_{i=1}^{3}\left(\operatorname{tgh}\left(\tilde{\zeta}_{i}\left(g, x^{5}\right)\right)\right)^{2}\right)^{-1 / 2} \frac{b_{1}\left(x^{5}\right)}{b_{0}\left(x^{5}\right)} \operatorname{tgh}\left(\tilde{\zeta}_{1}\left(g, x^{5}\right)\right)\right] x^{1}+ \\
-\left[\left(1-\sum_{i=1}^{3}\left(\operatorname{tgh}\left(\tilde{\zeta}_{i}\left(g, x^{5}\right)\right)\right)^{2}\right)^{-1 / 2} \frac{b_{2}\left(x^{5}\right)}{b_{0}\left(x^{5}\right)} \operatorname{tgh}\left(\tilde{\zeta}_{2}\left(g, x^{5}\right)\right)\right] x^{2}+ \\
-\left[\left(1-\sum_{i=1}^{3}\left(\operatorname{tgh}\left(\tilde{\zeta}_{i}\left(g, x^{5}\right)\right)\right)^{2}\right)^{-1 / 2} \frac{b_{3}\left(x^{5}\right)}{b_{0}\left(x^{5}\right)} \operatorname{tgh}\left(\tilde{\zeta}_{3}\left(g, x^{5}\right)\right)\right] x^{3}
\end{gathered}
$$




$$
\begin{aligned}
& \left(x^{\prime}\right)_{(g), D S R 4}^{1}\left(\{x\}_{m .}, x^{5}\right)=\left(x^{1}\right)_{(g), D S R 4}^{\prime}\left(\{x\}_{m .}, x^{5}\right)= \\
& =\sum_{\mu=0}^{3}\left(\exp \left(B_{(\rho(g), \widehat{v}(g) \equiv \widehat{\varepsilon}(g)), D S R 4}\left(x^{5}\right)\right)\right)_{\mu}^{1} x^{\mu}= \\
& =-\left[\left(1-\sum_{i=1}^{3}\left(\operatorname{tgh}\left(\tilde{\zeta}_{i}\left(g, x^{5}\right)\right)\right)^{2}\right)^{-1 / 2} \frac{b_{0}\left(x^{5}\right)}{b_{1}\left(x^{5}\right)} \operatorname{tgh}\left(\tilde{\zeta}_{1}\left(g, x^{5}\right)\right)\right] x^{0}+ \\
& +\left[1+\left(\left(1-\sum_{i=1}^{3}\left(\tilde{\zeta}_{i}\left(g, x^{5}\right)\right)^{2}\right)^{-1 / 2}-1\right) \frac{\left(\operatorname{tgh}\left(\tilde{\zeta}_{1}\left(g, x^{5}\right)\right)\right)^{2}}{\sum_{k=1}^{3}\left(\operatorname{tgh}\left(\tilde{\zeta}_{k}\left(g, x^{5}\right)\right)\right)^{2}}\right] x^{1}+ \\
& +\left[\left(\left(1-\sum_{i=1}^{3}\left(\operatorname{tgh}\left(\tilde{\zeta}_{i}\left(g, x^{5}\right)\right)\right)^{2}\right)^{-1 / 2}-1\right) \bullet\right. \\
& \left.\bullet \cdot \frac{b_{2}\left(x^{5}\right)}{b_{1}\left(x^{5}\right)} \frac{\left(\operatorname{tgh}\left(\tilde{\zeta}_{1}\left(g, x^{5}\right)\right)\right)\left(\operatorname{tgh}\left(\tilde{\zeta}_{2}\left(g, x^{5}\right)\right)\right)}{\sum_{k=1}^{3}\left(\operatorname{tgh}\left(\tilde{\zeta}_{k}\left(g, x^{5}\right)\right)\right)^{2}}\right] x^{2}+ \\
& +\left[\left(\left(1-\sum_{i=1}^{3}\left(\operatorname{tgh}\left(\tilde{\zeta}_{i}\left(g, x^{5}\right)\right)\right)^{2}\right)^{-1 / 2}-1\right) \bullet\right. \\
& \left.\bullet \frac{b_{3}\left(x^{5}\right)}{b_{1}\left(x^{5}\right)} \frac{\left(\operatorname{tgh}\left(\tilde{\zeta}_{1}\left(g, x^{5}\right)\right)\right)\left(\operatorname{tgh}\left(\tilde{\zeta}_{3}\left(g, x^{5}\right)\right)\right)}{\sum_{k=1}^{3}\left(\operatorname{tgh}\left(\tilde{\zeta}_{k}\left(g, x^{5}\right)\right)\right)^{2}}\right] x^{3}
\end{aligned}
$$




$$
\begin{aligned}
& \left(x^{\prime}\right)_{(g), D S R 4}^{2}\left(\{x\}_{m .}, x^{5}\right)=\left(x^{2}\right)_{(g), D S R 4}^{\prime}\left(\{x\}_{m .}, x^{5}\right)= \\
& =\sum_{\mu=0}^{3}\left(\exp \left(B_{(\rho(g), \widehat{v}(g) \equiv \widehat{\varepsilon}(g)), D S R 4}\left(x^{5}\right)\right)\right)_{\mu}^{2} x^{\mu}= \\
& =-\left[\left(1-\sum_{i=1}^{3}\left(\operatorname{tgh}\left(\tilde{\zeta}_{i}\left(g, x^{5}\right)\right)\right)^{2}\right)^{-1 / 2} \frac{b_{0}\left(x^{5}\right)}{b_{2}\left(x^{5}\right)} \operatorname{tgh}\left(\tilde{\zeta}_{2}\left(g, x^{5}\right)\right)\right] x^{0}+ \\
& +\left[\left(\left(1-\sum_{i=1}^{3}\left(\operatorname{tgh}\left(\tilde{\zeta}_{i}\left(g, x^{5}\right)\right)\right)^{2}\right)^{-1 / 2}-1\right) \bullet\right. \\
& \left.\bullet \frac{b_{1}\left(x^{5}\right)}{b_{2}\left(x^{5}\right)} \frac{\left(\operatorname{tgh}\left(\tilde{\zeta}_{1}\left(g, x^{5}\right)\right)\right)\left(\operatorname{tgh}\left(\tilde{\zeta}_{2}\left(g, x^{5}\right)\right)\right)}{\sum_{k=1}^{3}\left(\operatorname{tgh}\left(\tilde{\zeta}_{k}\left(g, x^{5}\right)\right)\right)^{2}}\right] x^{1}+ \\
& +\left[1+\left(\left(1-\sum_{i=1}^{3}\left(\operatorname{tgh}\left(\tilde{\zeta}_{i}\left(g, x^{5}\right)\right)\right)^{2}\right)^{-1 / 2}-1\right) \frac{\left(\operatorname{tgh}\left(\tilde{\zeta}_{2}\left(g, x^{5}\right)\right)\right)^{2}}{\sum_{k=1}^{3}\left(\operatorname{tgh}\left(\tilde{\zeta}_{k}\left(g, x^{5}\right)\right)\right)^{2}}\right] x^{2}+ \\
& +\left[\left(\left(1-\sum_{i=1}^{3}\left(\operatorname{tgh}\left(\tilde{\zeta}_{i}\left(g, x^{5}\right)\right)\right)^{2}\right)^{-1 / 2}-1\right) \bullet\right. \\
& \left.\bullet \frac{b_{3}\left(x^{5}\right)}{b_{2}\left(x^{5}\right)} \frac{\left(\operatorname{tgh}\left(\tilde{\zeta}_{2}\left(g, x^{5}\right)\right)\right)\left(\operatorname{tgh}\left(\tilde{\zeta}_{3}\left(g, x^{5}\right)\right)\right)}{\sum_{k=1}^{3}\left(\operatorname{tgh}\left(\tilde{\zeta}_{k}\left(g, x^{5}\right)\right)\right)^{2}}\right] x^{3}
\end{aligned}
$$




$$
\begin{aligned}
& \left(x^{\prime}\right)_{(g), D S R 4}^{3}\left(\{x\}_{m .}, x^{5}\right)=\left(x^{3}\right)_{(g), D S R 4}^{\prime}\left(\{x\}_{m .}, x^{5}\right)= \\
& =\sum_{\mu=0}^{3}\left(\exp \left(B_{(\rho(g), \widehat{v}(g) \equiv \widehat{\varepsilon}(g)), D S R 4}\left(x^{5}\right)\right)\right)_{\mu}^{3} x^{\mu}= \\
& =-\left[\left(1-\sum_{i=1}^{3}\left(\operatorname{tgh}\left(\tilde{\zeta}_{i}\left(g, x^{5}\right)\right)\right)^{2}\right)^{-1 / 2} \frac{b_{0}\left(x^{5}\right)}{b_{3}\left(x^{5}\right)} \operatorname{tgh}\left(\tilde{\zeta}_{3}\left(g, x^{5}\right)\right)\right] x^{0}+ \\
& +\left[\left(\left(1-\sum_{i=1}^{3}\left(\operatorname{tgh}\left(\tilde{\zeta}_{i}\left(g, x^{5}\right)\right)\right)^{2}\right)^{-1 / 2}-1\right) \bullet\right. \\
& \left.\bullet \frac{b_{1}\left(x^{5}\right)}{b_{3}\left(x^{5}\right)} \frac{\left(\operatorname{tgh}\left(\tilde{\zeta}_{1}\left(g, x^{5}\right)\right)\right)\left(\operatorname{tgh}\left(\tilde{\zeta}_{3}\left(g, x^{5}\right)\right)\right)}{\sum_{k=1}^{3}\left(\operatorname{tgh}\left(\tilde{\zeta}_{k}\left(g, x^{5}\right)\right)\right)^{2}}\right] x^{1}+ \\
& +\left[\left(\left(1-\sum_{i=1}^{3}\left(\operatorname{tgh}\left(\tilde{\zeta}_{i}\left(g, x^{5}\right)\right)\right)^{2}\right)^{-1 / 2}-1\right) \bullet\right. \\
& \left.\bullet \frac{b_{2}\left(x^{5}\right)}{b_{3}\left(x^{5}\right)} \frac{\left(\operatorname{tgh}\left(\tilde{\zeta}_{2}\left(g, x^{5}\right)\right)\right)\left(\operatorname{tgh}\left(\tilde{\zeta}_{3}\left(g, x^{5}\right)\right)\right)}{\sum_{k=1}^{3}\left(\operatorname{tgh}\left(\tilde{\zeta}_{k}\left(g, x^{5}\right)\right)\right)^{2}}\right] x^{2}+ \\
& +\left[1+\left(\left(1-\sum_{i=1}^{3}\left(\operatorname{tgh}\left(\tilde{\zeta}_{i}\left(g, x^{5}\right)\right)\right)^{2}\right)^{-1 / 2}-1\right) \frac{\left(\operatorname{tgh}\left(\tilde{\zeta}_{3}\left(g, x^{5}\right)\right)\right)^{2}}{\sum_{k=1}^{3}\left(\operatorname{tgh}\left(\tilde{\zeta}_{k}\left(g, x^{5}\right)\right)\right)^{2}}\right] x^{3} .
\end{aligned}
$$




\section{CONCLUSIONS}

In this paper, we discussed the finite structure of the space-time rotations in generalized Minkowski spaces $\widetilde{M_{N}}\left(\{x\}_{n . m .}\right)$, i.e. $N$-dimensional spaces endowed with a (in general non-diagonal) metric tensor, whose coefficients do depend on a set of non-metrical coordinates. We considered in detail (without loss of generality) the four-dimensional case. In particular, the results obtained have been specialized to the case of a "deformed" Minkowski space $\widetilde{M}_{4}$ (i.e. a pseudoeuclidean space with metric coefficients depending on energy), for which we derived the explicit general form of the finite rotations and boosts in different parametric bases.

This concludes the study (started in Ref. [1]) of the rotational component of the maximal Killing group of $\widetilde{M_{N}}\left(\{x\}_{n . m}\right.$. $)$, namely of the $N$-d. generalized, homogeneous Lorentz group $S O(T, S)_{G E N .}^{N(N-1) / 2}$, subgroup of the generalized

Poincaré group $P(S, T)_{G E N .}^{N(N+1) / 2}$. The space-time translations in generalized Minkowski spaces will be investigated in a forthcoming paper. 


\section{References}

[1] F. Cardone, A. Marrani, and R. Mignani, "Killing symmetries of generalized Minkowski spaces. 1-Algebraic-infinitesimal structure of space-time rotation groups", Found. Phys. 34, 617 (2004), hep-th/0505088.

[2] F. Cardone and R. Mignani, "On a nonlocal relativistic kinematics", INFN preprint n.910 (Roma, Nov. 1992); Grav. \& Cosm. 4, 311 (1998);

Found. Phys. 29, 1735 (1999); Ann. Fond. L. de Broglie 25, 165 (2000).

[3] F. Cardone, R. Mignani, and R.M. Santilli, J. Phys. G 18, L61, L141 (1992).

[4] F. Cardone and R. Mignani, JETP 83, 435 [Zh. Eksp. Teor. Fiz. 110, 793] (1996); F. Cardone, M. Gaspero, and R. Mignani, Eur. Phys. J. C 4, 705 (1998).

[5] F. Cardone and R. Mignani, Ann. Fond. L. de Broglie, 23 , 173 (1998);

F. Cardone, R. Mignani, and V.S. Olkhovski, J. de Phys.I (France) 7, 1211 (1997); Modern Phys. Lett. B 14, 109 (2000).

[6] F. Cardone and R. Mignani, Int. J. Modern Phys. A 14, 3799 (1999).

[7] F. Cardone, M. Francaviglia, and R. Mignani, Gen. Rel. Grav. 30, 1619 (1998); ibidem, 31, 1049 (1999); Found. Phys. Lett. 12, 281, 347 (1999).

[8] See e.g. F. Gursey: "Introduction to Group Theory", in Relativity, Groups and Topology, C. De Witt and B. De Witt eds. (Gordon and Breach, N.Y., 1964). 
[9] F. Cardone, A. Marrani, and R. Mignani, "Killing symmetries of generalized Minkowski spaces. 3-Space-time translations in four dimensions", Found. Phys. (submitted for publication).

[10] F. Cardone, A. Marrani, and R. Mignani, Found. Phys. Lett. 16, 163 (2003), hep-th/0505032.

[11] R. M. Santilli: Hadr. J. 8 25, 36 (1985).

[12] See e.g. N. Jacobson : Lie Algebras (Dover Publications, New York 1962); M.Naimark, A.Stern : Théorie des Représentations des groupes (Ed. Mir, Mosca 1979).

[13] A. Marrani: "Simmetrie di Killing di Spazi di Minkowski generalizzati" ("Killing Symmetries of Generalized Minkowski Spaces") (Laurea Thesis), Rome, October 2001 (in Italian). 